\title{
Review Article \\ Review on Recent Advances in Pulse Detonation Engines
}

\author{
K. M. Pandey and Pinku Debnath \\ Department of Mechanical Engineering, National Institute of Technology, Silchar, Assam 788010, India \\ Correspondence should be addressed to K. M. Pandey; kmpandey2001@yahoo.com
}

Received 27 July 2015; Revised 19 November 2015; Accepted 29 November 2015

Academic Editor: Sergey M. Frolov

Copyright (C) 2016 K. M. Pandey and P. Debnath. This is an open access article distributed under the Creative Commons Attribution License, which permits unrestricted use, distribution, and reproduction in any medium, provided the original work is properly cited.

\begin{abstract}
Pulse detonation engines (PDEs) are new exciting propulsion technologies for future propulsion applications. The operating cycles of PDE consist of fuel-air mixture, combustion, blowdown, and purging. The combustion process in pulse detonation engine is the most important phenomenon as it produces reliable and repeatable detonation waves. The detonation wave initiation in detonation tube in practical system is a combination of multistage combustion phenomena. Detonation combustion causes rapid burning of fuel-air mixture, which is a thousand times faster than deflagration mode of combustion process. PDE utilizes repetitive detonation wave to produce propulsion thrust. In the present paper, detailed review of various experimental studies and computational analysis addressing the detonation mode of combustion in pulse detonation engines are discussed. The effect of different parameters on the improvement of propulsion performance of pulse detonation engine has been presented in detail in this research paper. It is observed that the design of detonation wave flow path in detonation tube, ejectors at exit section of detonation tube, and operating parameters such as Mach numbers are mainly responsible for improving the propulsion performance of PDE. In the present review work, further scope of research in this area has also been suggested.
\end{abstract}

\section{Introduction}

In present days the attention of researchers in propulsion field from all over the world has turned towards the pulse detonation engine historical background, thermodynamics analysis, detonation initiation, and deflagration to detonation wave transition device as their main subject in detonation combustion research area. Another review research on rotating detonation engine model and application on aerospace and turbomachinery and performance are also included in this area. These involve researches from the United States, Russia, Japan and China, Germany, and Malaysia. Numbers of research publications have increased significantly in the past few decades. The main attraction of detonation combustion was to generate shock wave that is followed by combustion wave [1]. Pratt and Whitney began to develop the pulse detonation engine in 1993. Their research approach was to study the deflagration to detonation transition through the pulse detonation engine [2]. The feasibility study of a reaction device operating on intermittent gaseous detonation wave is considered by Nicholls et al. [3]. They conducted a study to investigate the thrust, fuel flow, air flow, and temperature over the range of operating conditions. Recently many countries give much importance to research of multimode combined detonation engine in hypersonic aircrafts propulsion system [4]. Kailasanath [5] studied a review on practical implementation on pulse detonation engine and deflagration to detonation transition was also studied in obstacle geometry. Again Kailasanath studied the development of pulse detonation engine. The detonation combustion parameters such as Chapman velocity and pressure are well derived in this study [6]. Wilson and Lu [7] summarized integrated studies for both PDE and RDE based propulsion system. They focused detonation waves to hypersonic flow simulation and power generation. Smirnov et al. [8] studied numerical simulation of detonation engine fed by fuel-oxygen mixture. The advantage of a constant volume combustion cycle as compared to constant pressure combustion was in terms of thermodynamic efficiency focused for advanced propulsion on detonation engine.

\section{Reviews on Experimental Analysis}

Chen et al. [9] investigated experimentally the nozzle effects on thrust and inlet pressure of an air-breathing pulse detonation engine. Their results showed that thrust augmentation 
of converging-diverging nozzle, diverging nozzle, or straight nozzle is better than that of converging nozzle on whole operating conditions. Li et al. [10] conducted an experiment on PDRE model utilizing kerosene as the fuel, oxygen as oxidizer, and nitrogen as purge gas. The thrust and specific impulse were investigated experimentally. Their obtained results showed that the thrust of PDRE test model was approximately proportional to detonation frequency. The time average thrust was around $107 \mathrm{~N}$. Yan et al. [11] studied the performance of pulse detonation engine with bell-shaped convergent-divergent nozzle. This experiment has been done using kerosene as liquid fuel, oxygen as oxidizer, and nitrogen as purge gas. Their tested result has shown that the maximum thrust augmentation is approximately $21 \%$. Allgood et al. [12] experimentally measured the damped thrust of multicycle pulse detonation engine with exhaust nozzle. Their results showed that diverging nozzle increases the performance with increase in fill fraction. Peng et al. [13] studied the two-phase dual-tube air-breathing pulse detonation engine (APDE) experiments to improve the understanding of characteristics of valveless multitube APDE. From the experimental results it is seen that the comparison between single- and dual-tube firing and the operation pattern of single-tube firing is beneficial to reduce the disturbance in the common air inlet. Yan et al. [14] experimentally investigated pulse detonation rocket engine with injectors and nozzles. The injectors were tested for atomization and mixing of two phase reactants. They observed that nozzles are the critical component for improving the performance of PDE. From their tested results they observed that a nozzle with high contraction ratio and high expansion ratio generated the highest thrust augmentation of 27.3\%. Kasahara et al. [15] tested "Todoroki" rocket system at different operating conditions. The maximum thrust was produced slightly above $70 \mathrm{~N}$ with a specific impulse up to $232 \mathrm{~s}$. The frequency of the system, even for a constant supply rate of propellants, varied over the range of $40-160 \mathrm{~Hz}$. Copper et al. [16] measured impulse by using a Ballistic pendulum arrangement for detonation and deflagration in a tube closed at one end. They also studied the effect of internal obstacles on the transition from deflagration to detonation (see Figure 6). Their experimental results and prediction from analytical model are agreed within $15 \%$. Hinkey et al. [17] experimentally demonstrated a rotary valve pulse detonation engine combustor for high frequency operation. Their experiments series were conducted in rotary valve single combustor and rotary valve multicombustor pulse detonation engine. The main measuring parameters are thrust as well as combustor wall pressure histories, oxidizer, and fuel mass flow rate (see Figure 14). Their concept of system operation was successfully demonstrated in rotary valve multicombustor PDE. In Japan, recently a single-tube pulse detonation rocket system that can slide on rails was fabricated. In the sliding test, the system operated 13 cycles at the frequency of $6.67 \mathrm{~Hz}$ [18]. Li et al. [19] illustrated the detonation initiation area in a detonation chamber. This detonation tube was closed at one end and open at another, which was composed of thrust wall and ignition section. The three spiraling internal grooves like semicircle, square, and inversed-triangle grooves were used in this experimentation. The results showed that the spiraling internal groove can effectively enhance DDT. Asato et al. [20] have studied experimentally the effects of rapid flame propagation, rotating velocity, and Shchelkin spiral dimension in the vortex flow on DDT characteristics. The vortex flow was generated and that was promoted by Shchelkin spiral dimensions and DDT distance in the vortex flow could be shortened by $50-57 \%$. New et al. [21] experimentally investigated Shchelkin spiral effect on multicycle pulse detonation engine. The effectiveness of Shchelkin spiral parameters on DDT phenomenon was studied using propane-oxygen mixture at low energy ignition source. The various configurations like spiral blockage ratio and spiral length to diameter ratio were also studied. In these studies shorter length configurations and highest blockage ratio were successful and sustained DDT was achieved. Wang et al. [22] performed the numbers of experiments on spiral configuration in pulse detonation engine. Their analysis provided the design data for deflagration to detonation transition rule in curved detonation chamber. Some experiments have been conducted using nine tubes in resistance experiments and result indicates that there is no detonation wave formed in the straight tube, but fully developed detonation waves have been obtained at selected spiral tubes. Panicker et al. [23] studied specific techniques for deflagration to detonation transition that were considered including Shchelkin spirals, grooves, converging-diverging nozzles, and orifice plates. They observed that Shchelkin spiral is to be the best performer for deflagration to detonation wave transition among other DDT enhancement devices.

Valiev et al. [24] investigated the "Flame Acceleration in Channels with Obstacles in the Deflagration-to-Detonation Transition." They found that obstacle mechanism is much stronger to accelerate deflagration flame to detonation wave. The physical mechanism of deflagration flame acceleration in an obstacle channel is quite different from Shchelkin spiral mechanism. The obstacle mechanism is much stronger for deflagration to detonation wave transition and it depends on operating conditions. The mechanism of viscous heating was also identified with proper modifications of obstacle geometry. Gaathaug et al. [25] numerically studied the deflagration to detonation transition in a turbulent jet behind an obstacle. The spiral internal grooves and inversed-triangle grooves were tested to enhance DDT and results showed that the spiraling internal groove can effectively enhance DDT. Moen et al. [26] studied the influence of obstacles on propagation of cylindrical flame. The freely expanding cylindrical type flame speed depends on obstacle configuration and achieved appropriate turbulence in obstructed flame travel path.

Ogawa et al. [27] studied flame acceleration and DDT in square obstacle array by solving Navier-Stokes equations. The computational simulation shows that deflagration wave acceleration was effected by obstacle series. Johansen and Ciccarelli [28] investigated the effect of obstacle blockage ratio on development of unburnt gas flow field for varying obstacle height. The computational simulations show that turbulence production increases with increasing number of blockage. Gamezo et al. [29] studied numerically deflagration wave acceleration and deflagration to detonation transition in obstructed channels. From the simulation they observed that detonation is ignited when a Mach stem formed by the 


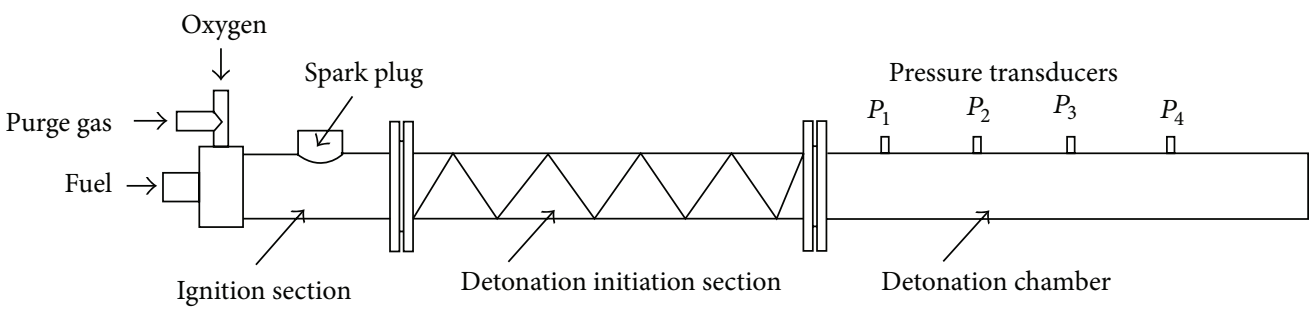

FIgURE 1: Schematic diagram of detonation tube with spiral [19].
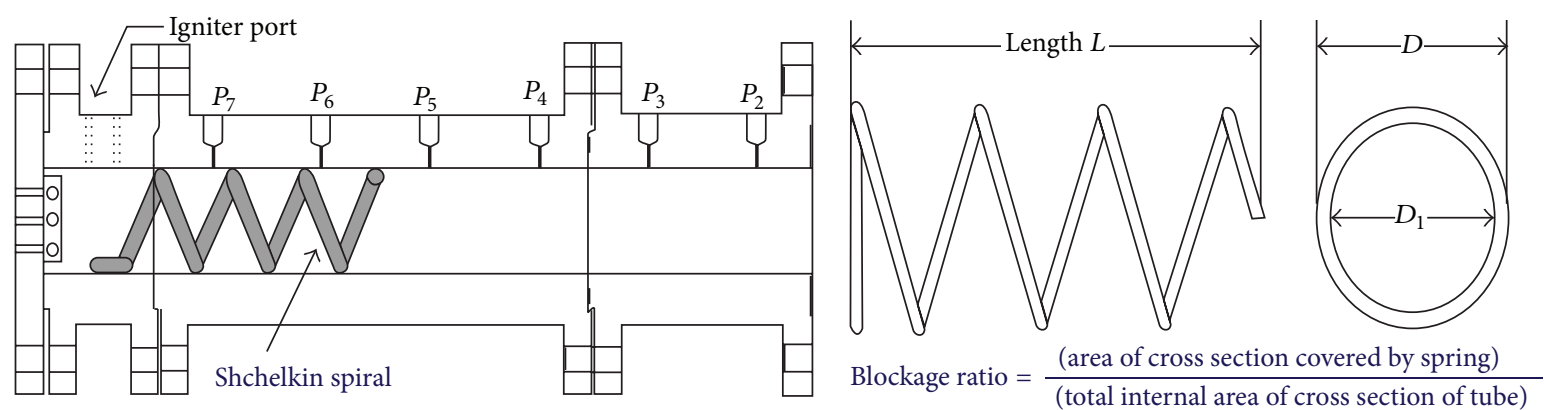

Figure 2: Schematic view of Shchelkin spiral configuration [21].

diffracting shock reflecting from the side wall collides with an obstacle. Johansen and Ciccarelli [30] studied the effect of obstacle blockage ratio on the development of unburnt gas flow field ahead of flame front in an obstacle channel using large eddy simulation. The simulations indicate that turbulence production increases with increasing number of obstacles. The quasi-detonation regime is characterized by an average flame velocity that significantly depends on the geometry of detonation tube (see Figure 1) [31]. A series of high frequency detonation wave tests is conducted by Huang et al. [32] in small scale pulse detonation engine using keroseneair as the fuel-oxidizer to seek DDT enhancement efficiency. They observed that DDT distance and transition time were reduced. Rudy et al. [33] studied that the flame acceleration in obstructed channel has important applications for supersonic propulsion technology. The mechanism of DDT in hydrogenair mixtures was experimentally investigated in obstacle channel using pressure profiles, wave velocities, and numerical calculations. Their results also show that obstacle blockage ratio and spacing have strong influence on detonation wave velocity stability. A weak thrust experiment on pulse detonation engine was conducted by Baklanov et al. [34]. The test was done to perform the oxidant on engine operation mode influence of ring obstacle on deflagration to detonation transition. Air-hydrogen and air-hydrocarbon mixture was used in this test.

Ciccarelli et al. [42] studied experimentally the effect of obstacle blockage on the rate of flame acceleration and on the final quasi-steady flame-tip velocity. In a smooth tube detonation transition occurs when the flame acceleration eventually leads to a terminal velocity under $1000 \mathrm{~m} / \mathrm{s}$. The freely expanding cylindrical flame speed depends on obstacle configuration and achieved appropriate turbulence in obstacles flame flow path. Gamezo et al. [43] experimentally investigated

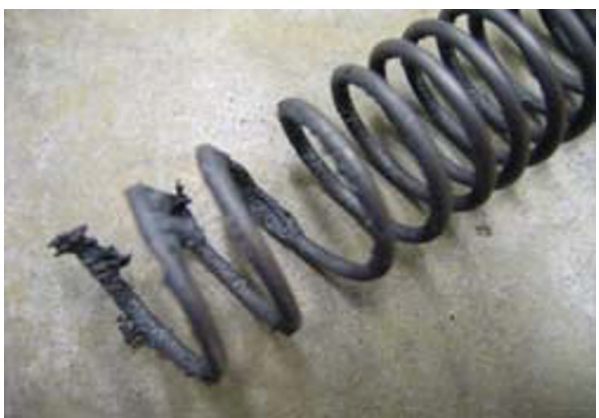

FIGURE 3: Photographs of experimental Shchelkin spiral [23].

the flame acceleration and DDT in hydrogen-air mixture in obstacle channel using $2 \mathrm{D}$ and $3 \mathrm{D}$ reactive Navier-Stokes numerical simulations and observed the regimes of supersonic turbulent flames, quasi-detonation, and detonation flame propagation behind the leading shock wave. Johansen and Ciccarelli [44] studied the effect of obstacle blockage ratio on development of unburnt gas flow field using varying obstacle height. The effect of blockage ratio on flame acceleration was investigated in an obstructed square cross section channel. Paxson [35] developed a simple computational code to access the impact of DDT (see Figure 8) enhancing obstacles on pulse detonation engine (see Figures 4 and 7). The simulation was to examine the relative contributions from drag and heat transfer. Pulse detonation engine observed that heat transfer is more significant than aerodynamic drag. Frolov [36] studied the deflagration to detonation transition in gas and drop air-fuel mixture. In this study reflecting elements could improve the fast deflagration to detonation transition of kerosene-air mixture. Teodorczyk [37] studied the flame 


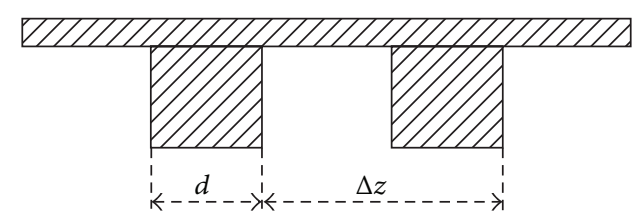

(a)

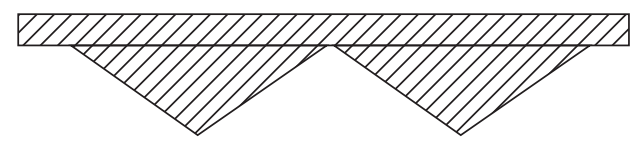

(b)

FIGURE 4: Schematic view of typical obstacle shape discussed in ICDERS-2007 [24].

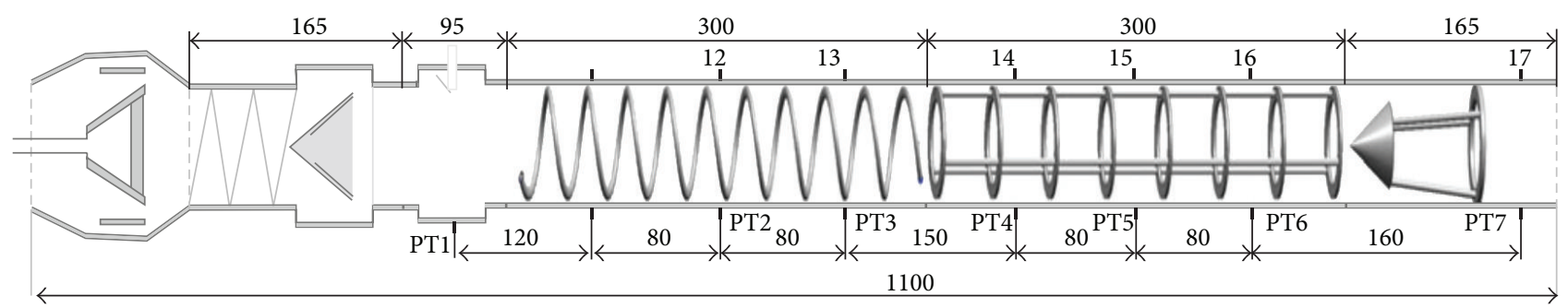

FIGURE 5: Schematic view of the small scale pulse detonation engine test setup with Shchelkin spiral and obstacle blockage [32].

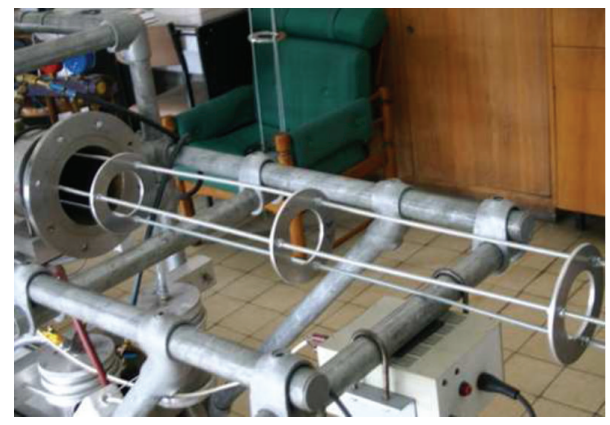

FIGURE 6: Experimental setup of obstacles location along the detonation tube [33].

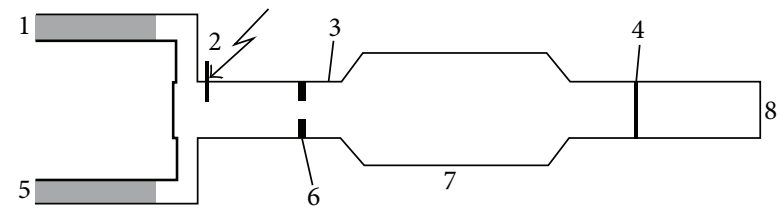

FIGURE 7: Schematic view of pulse detonation engine: 1 and 5: fuel and oxidant feed manifolds, 2: spark plug, 3: combustion chamber, 4: detonation wave, 6: ring obstacle, 7: prechamber, and 8: nozzle [34].

propagation using channel heights of $0.01,0.02,0.04$, and $0.08 \mathrm{~m}$ experimentally. The velocities of flame propagation in the obstructed channel were established in this study. The experimental result was found that deflagration to detonation transition and flame propagation regimes were established.

Frolo et al. [45] studied detonation characteristics in a Ubend tube to simulate DDT and their analysis concluded that U-bend is useful for quicker DDT. Frolov et al. [38] studied shock to detonation transition in U-bend tube experimentally and computationally both. The simulation results demonstrated a considerable effect of U-bend tube on detonation
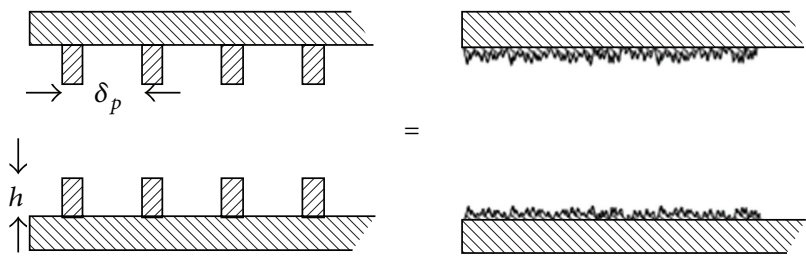

Figure 8: Schematic view of DDT obstacle layout [35].

initiation. Semenov et al. [46] proposed the parabolic contraction and conical expansion for detonation initiation in a tube. The shine shaped wall is proposed for optimized geometry of conical expansion. They observed that the minimal incident shock wave velocity of $680 \pm 20 \mathrm{~m} / \mathrm{s}$ is approximately Mach number of 2. The U-bend is used to optimize the design of pulse detonation engine by Frolov et al. [39]. The numerical simulation of this optimization process reveals some features of deflagration to detonation transition in U-bend tubes.

Ejector is a device which is placed downstream of the pulse detonation combustor exit, coaxial the detonation tube, and it is used for propulsion performance implementation. Allgood and Gutmark [47] provided two-dimensional ejectors to pulse detonation engine for parametric study and performance was observed for inlet geometry and axial position relative to the exhaust section of PDE. Yan et al. [48] conducted an experiment in a small scale pulse detonation rocket engine (see Figure 5) which was used as a predetonator to initiate detonation in its ejectors. In this experiment they observed that flame propagation upstream at the entrance of the ejector was inevitable, which affected the detonation initiation process in the ejector. Another experimental study was performed by Bai and Weng [49] for investigating the ejector's effect on the performance of a pulse detonation engine. Their results indicated that thrust augmentation increases at high operating frequency. Canteins et al. [50] experimentally as well as numerically observed that performances of PDE 


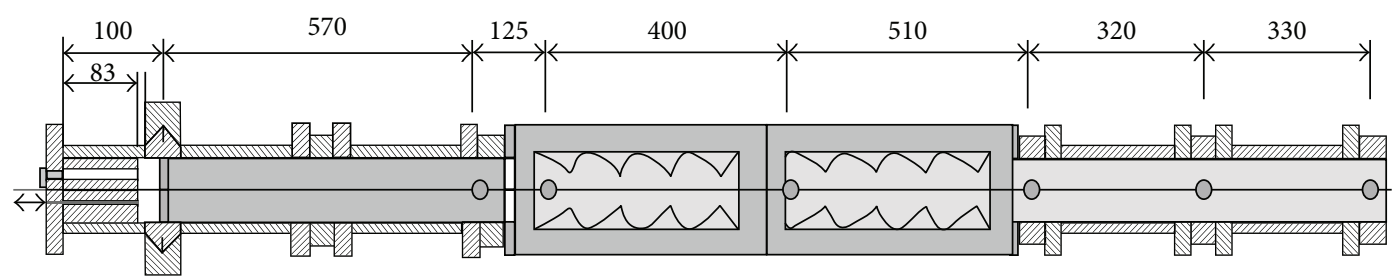

FIGURE 9: Scheme view of experimental unit with profiled regular obstacles [36].

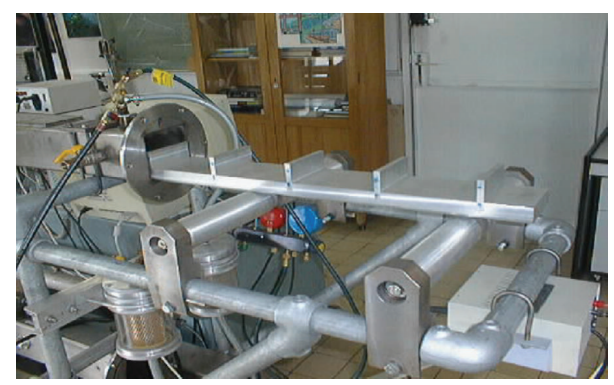

Figure 10: The photo of the channel with obstacles [37].

are varied with three geometry parameters of the ejector, that is, inner diameter, ejector length, and ejector position relative to the thrust wall of combustion chamber. For these configurations an ejector increases the specific impulse up to $60 \%$. Cha et al. [51] suggested combined effect of bypass and ejector concept on the analyses of air-breathing pulse detonation engine. The calculated results showed that the APDE performance is determined by shock loss induced by throat and diameter of the nozzle. The experiments were also designed by Santoro et al. [52] to probe different aspects of PDE/ejector setup. The results indicate that, for the geometries studies, the maximum thrust augmentation of $24 \%$ is achieved. Linear array and compact box array of detonation tube with axisymmetric ejectors were examined by Hoke et al. [53]. In this study secondary flow was engaged with the ejector lip by linear detonation tube. Further the thrust augmentation was found as a function of ejector entrance to detonation tube exit distance. This thrust augmentation factor of 2.5 was observed using a tapered ejector and it was also observed that thrust augmentation depends on the distance between the detonation tube exit and the ejector entrance. The maximum thrust depends on both positive and negative values of this distance [54]. The tapered ejector and cylindrical ejector configurations were tested by Paxson et al. [55] for thrust augmentation. From this test desired results are achieved in tapered ejector configuration. Glaser et al. [56] conducted an experiment on the operation of pulse detonation engine-driven ejectors. The experimental studies employed an $\mathrm{H}_{2}$-air mixture in PDE with ejectors to improve the performance augmenters driven. In this research straight and diverging ejectors were investigated. The optimum axial placement was found to be downstream of the pulse detonation engine. To improve the performance of ejector driven by an air-breathing pulse detonation engine the experiments were carried out with convergent nozzle at different operating frequencies by Changxin et al. [57]. The maximum thrust augmentation was obtained in singlestage ejector for $L / D=2$. Korobov and Golovastov [58] studied the ejector influence effectiveness of a detonation engine and their results showed that the use of an ejector can yield thrust augmentation by $17 \%$. Huang et al. [59] experimentally studied the noise radiation characteristics of multicycle pulse detonation engine with and without ejectors. The results implied that the pulse sound pressure level increased with the increase in operating frequency. But the ejectors system showed that ejectors could decrease the peak sound pressure level of pulse detonation engine. Qiu et al. [60] conducted the operating characteristics of aerodynamic valves for pulse detonation engine for adaptive control of fuel. Their experimental results showed that residual fuel droplets moved upstream with backflow followed by flame and flame could propagate across the valve.

Matsuoka et al. [61] developed rotary valve for pulse detonation engine to analyze its basic characteristics and performance and they obtained maximum time averaged thrust of 71 N. Again Matsuoka et al. [62] developed the liquid-purge method as a novel approach to a pulse detonation combustor and they observed that detonations were successfully initiated by this method. Fan et al. [63] experimentally investigated the mixing and ignition characteristics of fully developed detonation in pulse detonation engine. The experimental results show that duty cycles should be kept as close as possible to attain effective mixing. Frolov et al. [64] conducted a low frequency demonstrator liquid fuel pulse detonation engine and their results reported that DDT occurs within a very short distance. An experimental study was investigated by Tangirala et al. [65] in multitube PDC-turbine hybrid system. The turbine component efficiency was found to be similar under PDC-fired operation. A series of experiments were carried out by $\mathrm{Li}$ et al. [66] on a pulse detonation engine using liquid kerosene-oxygen mixture to investigate the detonation wave initiation. The successful detonation wave was achieved when length of the spiral (see Figure 11) was increased six times of inner diameter of detonation tube. Stevens et al. [67] experimentally investigated that detonation produces shock strength and separation distance between shock and decoupled flame. Their observations indicated that higher Mach number is favored when attempting to reinitiate detonation on a reflecting ramp. Fan et al. [68] experimentally investigated on two-phase pulse detonation engine and their obtained results have demonstrated that the averaged thrust of PDE is approximately proportional to the volume of detonation chamber and detonation frequency. 


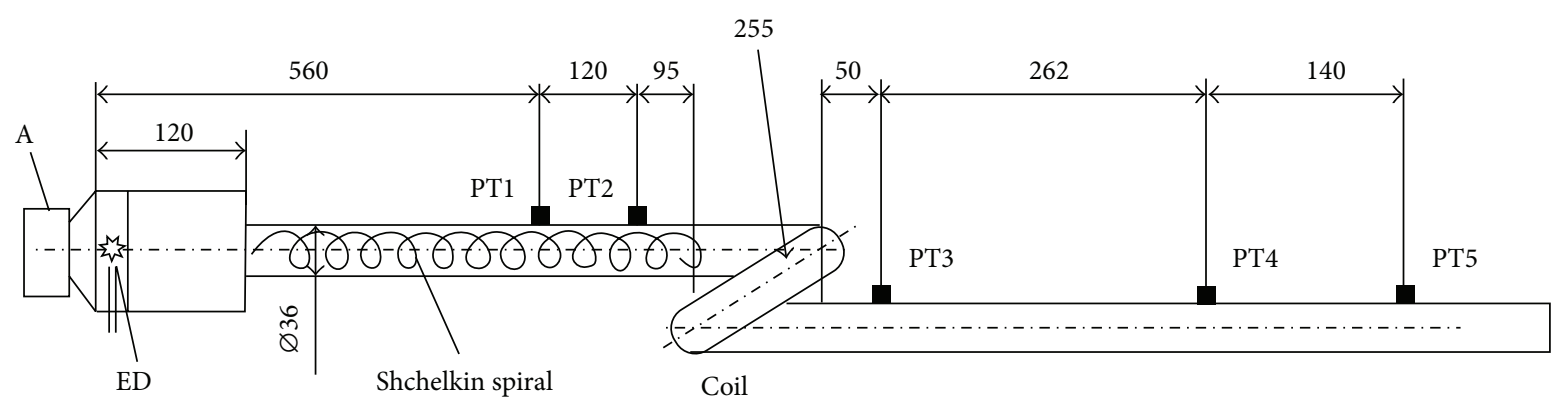

FIGURE 11: Schematic diagram of Shchelkin spiral and a coil inside the detonation tube [38].

The effects of small perturbations with varying blockage ratio on the critical tube diameter problems are investigated by Mehrjoo et al. [69]. They found that optimal blockage ratio is approximately $8 \%$ to $10 \%$ and suggested that it can be useful for design application of pulse detonation engines propulsion and power system. Huang et al. [70] conducted an experiment using liquid kerosene fuel and they observed heating liquid kerosene enhancing the engine performance, which was helpful for deflagration to detonation transition process. The experimental investigation was conducted by Deng et al. [71] to examine the operability of turbo machinery that a single stage radial flow turbine of a turbocharger in pulse detonation combustor operates. Their experimental results showed that the experimental rig can operate stably under the frequency up to $10 \mathrm{~Hz}$. Cha et al. [72] developed pulse temperature and stream concentration test device to measure the temperature and steam concentration at the exhaust plum of a pulse detonation engine. Their experimental results demonstrate that the frequency of the temperature and pressure is the same and their device is able to capture every pulse detonation quickly and precisely. Thrust measurement experiment was carried out by Morozumi et al. [73] using a four-cylinder pulse detonation engine with rotary valve. Their experimental results achieved time averaged thrust of $258.5 \mathrm{~N}$ and specific impulse of $138.7 \mathrm{~s}$. The detailed reactive detonation combustion flow features are experimentally investigated by Zitoun and Desbordes [74]. They observed that length to diameter ratio of combustion chamber is most important for thrust and specific impulse deficit. Fan et al. [75] conducted an experiment to investigate the effects of fuel preheating and adding additives on the detonation initiation time. From this analysis they achieved adding the additives to liquid kerosene, the detonation initiation time was reduced from $0.75 \mathrm{~ms}$ to $0.34 \mathrm{~ms}$.

\section{Reviews on Computational Analysis}

Experimental analysis in pulse detonation engine is needed to have observations carefully on detonation combustion simulations; some researchers observed that at the same time numerical simulations are equally important to visualize the detonation combustion phenomena in PDE combustor. The PDE combustor with and without obstacles having hydrogenair mixture has been simulated by Soni et al. [76] using a commercially available CFD code. They observed that obstacles are useful for PDE combustor design and development. Amin et al. [77] studied the effects of various nozzle geometries and operating conditions on the performance of a pulse detonation engine. The CFD results indicate that a diverging nozzle is more effective than a converging-diverging nozzle at low ambient pressure. Tangirala et al. [78] simulated the performance of PDE in subsonic and supersonic flight conditions. Their first parametric studies were employed in 2D CFD model. The results indicated that the exit nozzle enhances thrust generation, maintains operating pressure, and also controls operating frequency. Shao et al. [79] studied the effect of different types of nozzle on continuous detonation engine (CDE) using one step 3D numerical chemical reaction model. The analyzed four types of nozzles are, namely, the constant-area nozzle, Laval nozzle, diverging nozzle, and converging nozzle. The results indicated that Laval nozzle has great scope to improve the propulsion performance of this system. Ma et al. [80] studied the comparison between singletube and multitube PDEs and influence on the nozzle flow field to improve propulsive performance. In this study thrust variation in transverse direction occurred due to reduction in axial-flow oscillations and offers a wider operation range in terms of valve timing. They showed that convergent nozzle helps to keep the original chamber pressure constant and consequently improves the engine net performance. Wintenberger and Shepherd [81] studied the flow path in a singletube pulse detonation engine. They developed this analytical model for simulating the flow and for estimating the performance and they compared it with ideal ramjet engine. They observed that engine thrust depends on detonation tube impulse, momentum, and pressure terms. The result also indicated that the total pressure losses were caused due to unsteadiness of the flow. Kailasanath and Patnaik [82] presented a review of computational studies on pulse detonation engine. In this paper their objective was to evaluate the time dependent numerical simulations of PDE. They observed that the initial conditions in simulations are the significant effect on overall performance. Ma et al. [83] numerically studied internal flow dynamics of pulse detonation engine, which used ethylene as a combustible fuel. The detonation wave dynamics and flow evaluation were simulated. For the engine design optimization sensitivity study of operation time was also conducted. Frolov and Aksenov [84] demonstrated the deflagration to detonation transition in a tube with continuous flow of a prevaporized TS-1 jet kerosene-air mixture at 


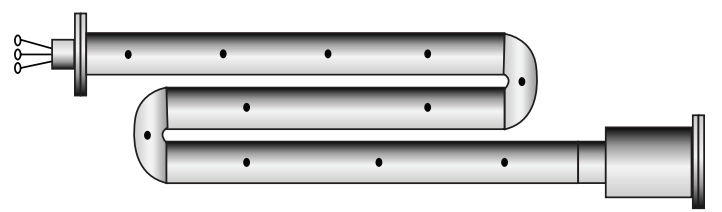

FIGURE 12: Experimental setup of U-bend tube indicates the pressure transducer location [39].

atmospheric pressure. In this analysis fuel combustion was observed in detonation combustion mode. Johansen and Ciccarelli [85] conducted large eddy simulation of initial flame acceleration in an obstructed channel (see Figure 10). The effect of obstacle blockage ratio on development of unburnt gas flow field ahead of flame front in an obstacle channel was investigated using large eddy simulation. The computational simulations indicate that turbulence production increases with increasing obstacle blockage ratio. The static thrust is easily achievable through a Lockwood-Hiller design, with a U-shaped configuration. This design needed to be tested for detonation wave simulation [40]. Again Frolov et al. [86] performed the detonation wave propagation in planar channel and cylindrical tube with two U-shaped bends of limiting curvature. Otsuka et al. [87] studied the influence of U-bend (see Figure 12) on detonation wave propagation with computational fluid dynamics analysis. Their results show that detonation waves disappear near the U-bend curvature inlet and restart after passing through it and also found that the U-bend with small channel width and curvature radius can induce fast DDT. The starting vortex was generated and performance was affected by the geometry of ejectors. On the other side ejector length is less important on overall performance compared to the ejector diameter [41]. The unsteady thrust augmentation was studied by Zheng et al. [88]. The optimum starting vortex was generated by optimum diameter of ejector and investigation also showed a minor effect of ejector length. The thrust augmentation was found to increase with ejector length. The ejector performance was observed to be strongly dependent on the operating fill fraction [89]. Zhang et al. [90] investigated the ejectors performance on thrust augmentation in pulse detonation engine. The numerical results were describing the primary detonation wave propagation processes of PDE and secondary detonation wave propagation in ejector system. The details of flow field of detonation wave propagation inside the detonation tube and injection into the ejectors were studied. The rotary wave ejector concept has highly significant potential for thrust augmentation relative to a basic pulse detonation engine [91]. Yi et al. [92] studied the effect of ejector in pulse detonation rocket propulsion system. They observed various features including detonationshock interaction, detonation diffraction, and vortex formation in propagation of hydrogen-oxygen mixture detonation. Stoddard et al. [93] studied the CFD analysis on detonation wave propagation and resulting exhaust gas dynamics. The simulations are taken towards optimizing a static thrust on pulse detonation engine.

Tan et al. [94] studied the detonation wave on film dynamics and concluded that thermal protection is very important and necessary for the design of a pulse detonation combustor. $\mathrm{He}$ and Karagozian [95] studied the transient, reactive compressible flow phenomena in pulse detonation wave engine computationally. The engine performance parameter and in addition engine noise were estimated within and external to detonation tube. Yungster et al. [96] studied numerically the formation of $\mathrm{NO}_{x}$ in hydrogen-fuelled pulse detonation engine. Their results indicated that $\mathrm{NO}_{x}$ formation in pulse detonation engine is very high for stoichiometric mixture. $\mathrm{Ma}$ et al. [83] studied numerically internal flow dynamics in a valveless air-breathing pulse detonation engine operating on ethylene fuel. Their calculated pressure histories and gross specific impulse of $1215 \mathrm{~s}$ show good agreement with experimental results.

\section{Performance Analysis on Detonation Combustion}

Historically, exergy analysis was developed to evaluate the process of power extraction from heat energy. The applications of exergy analysis for the performance assessment of power generation cycles have increased in recent years [97]. The exergy analysis gives a quantitative theoretical useful work that is obtained from different energy form in combustion process and is a function of the system and environment. The exergy of a fluid stream depends on reference environment [98]. Rouboa et al. [99] studied the exergy losses during the shock and rarefaction wave of hydrogen-air mixture. Their main objectives were the exergetic efficiency analysis using $1.5 \%, 2.5 \%$, and $5 \%$ hydrogen mass fraction in combustible air. They obtained exergetic efficiency of $77.2 \%$, $73.4 \%$, and $69.7 \%$ for aforesaid hydrogen concentrations. Bellini and Lu [100] studied the efficiency in terms of availability of power device operated by detonation wave. The second law or exergetic efficiency was computed based on the fuel availability and results are then compared with the exergetic efficiency applied to deflagration system. The exergy analysis makes clear that this device is efficient for power generation. Hutchins and Metghalchi [101] have performed energy and exergy analysis of the pulse detonation engine. Different fuels such as methane and jet propulsion (JP10) (see Figure 13) have been used in this analysis. The exergetic efficiency has been calculated in pulse detonation engine and simple gas turbine engine. The compared result shows that for same pressure ratio pulse detonation engine has better efficiency and effectiveness than gas turbine system. Bellini and $\mathrm{Lu}$ [102] studied the exergy analysis in pulse detonation power device which is designed for power production using methane $\left(\mathrm{CH}_{4}\right)$ and propane $\left(\mathrm{C}_{3} \mathrm{H}_{8}\right)$. The exergetic efficiency was studied for different design parameters like cycle frequency and detonation tube length. The theoretical analysis of pulse detonation engine over gas turbine has been carried out for electric power production. Safari et al. [103] studied that the transport equation of entropy is introduced in large eddy simulation to perform exergy analysis of turbulent combustion systems. In this methodology, the effects of chemical reaction and entropy generation contribution appear in closed forms. Fuel exergy of hydrogen was analyzed by Wu et al. [104] and computational results indicate that fuel 


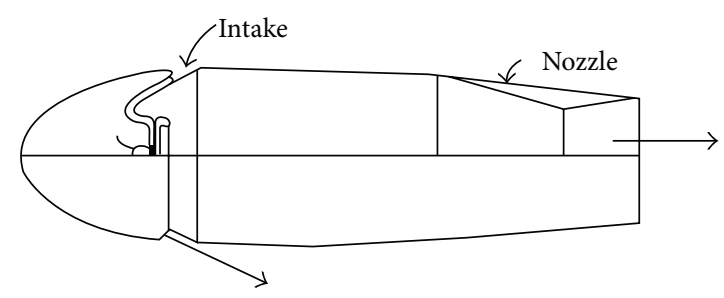

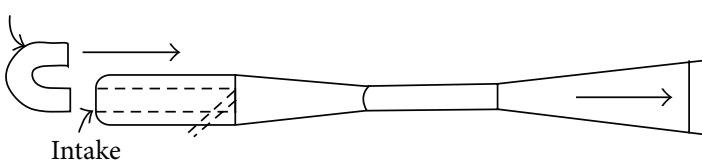

(a)

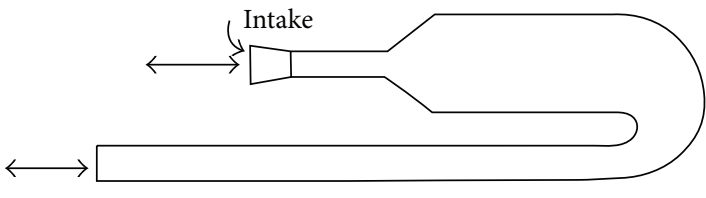

(b)

FIgURE 13: Non-PDE pulse jet designs and valveless pulsejet configurations [40].

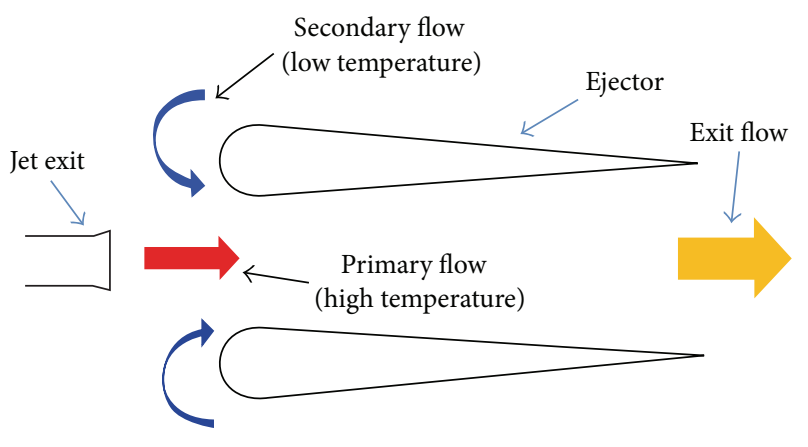

FIGURE 14: Combustion product mass flows through the ejectors [41].

exergy of hydrogen exceeded the lower heating value (LHV) and was less than the higher heating value (HHV). The fuelair mixture is ignited by high energy, high frequency source in combustion chamber. Hu et al. [105] studied that the fluid viscosity is a significant factor resulting in the energy loss in most fluid dynamical systems. The efficiency of PDE is affected by fuel viscosity due to exergy losses in pulse detonation engine. The energy loss in the Burgers model excited by periodic impulses is investigated based on the generalized multisymplectic method. Som and Sharma [106] developed the theoretical model of energy and exergy balance in a spray combustion process in a gas turbine combustor at various operating conditions. They observed that the velocity, temperature, and pressure field of the combustor are required for evaluating the flow availabilities and process irreversibility from a two-phase separated flow model of spray combustion.

Theoretical analysis of pulse detonation engine was estimated by Endo and Fujiwara [107]. The time average thrust was estimated from their simple theoretical model. Zel'dovich [108] studied the detonation combustion products calculation using Jouguet theory. The products of combustion are quite high in kinetic energy as well as heat energy and their statement is in good agreement with experimental analysis. Heiser and Pratt [109] studied thermodynamic cycle analysis of pulse detonation engine and their results show that below Mach 3 performance of PDE cycle is better than real Brayton cycle. Wu et al. [110] studied the propulsive efficiency of pulse detonation engine and system performance in terms of thrust and specific impulse and, also, compared with gas turbine and ramjet engines. Li and Kailasanath [111] studied that the flow field pressure wave developed in pulse detonation engine, which is responsible for thrust production. Cooper et al. [112] studied the specific impulse over the Mach number regime. Their analysis represents that the specific impulse varies with Mach number up to a certain limit; after that it does not change with Mach number increases. Schauer et al. [113] studied the research efforts on detonation initiation, propagation, heat transfer, and other parameters for the performance analysis.

\section{Reviews on Detonation Combustion}

In the 1970s a substantial effort was undertaken to investigate the feasibility of using detonative propulsion for thrusters in the dense or high-pressure atmospheres of solar system planets [114]. In subsonic flow condition the combustion of propellant in longitudinal pulsed and spin detonation waves is experimentally studied by Bykovskii and Vedernikov [115] and they observed that stability of detonation regimes depends on counter pressure of the medium. Detonation combustion produces higher rate of energy release compared to the deflagration combustion. The rate of energy release in detonation mode of combustion normally depends on the magnitudes of deflagration flame [116]. The fact that contributes to the research is more towards the gaseous fuel rather than liquid fuels which need an additional system for PDE, which evaporate the liquid fuel to gaseous state before it can get detonated. However liquid fuels have advantages over the gaseous fuel as it is best suitable for the volume limited aerospace system and at high altitude applications [117]. Radulescu and Hanson [118] studied the convictive heat losses in reactive flow field in pulse detonation engine. They showed that nondimensional tube length $L / D$ ratio, that is, length to the diameter ratio, governs the amount of heat losses. Shock 


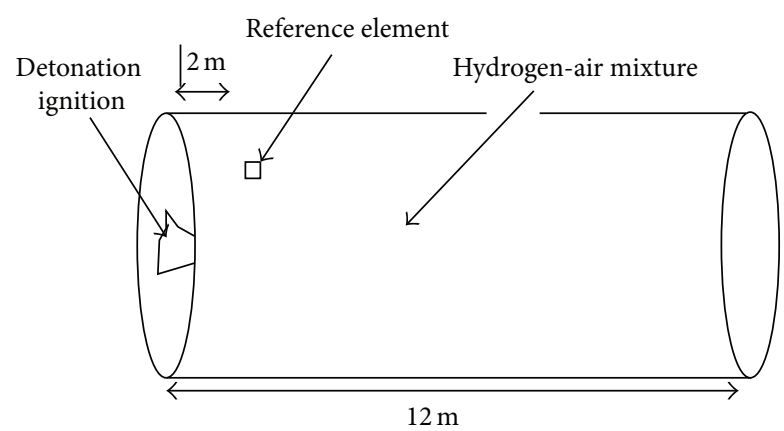

FIGURE 15: Schematic view of shock tube for exergy analysis [99].

wave propagation in a detonation tube of pulse detonation engine was investigated experimentally by Driscoll et al. [119]. Their results indicated that crossover length of detonation tube is directly related to strength of transfer shock in detonation tube. Li et al. [120] studied detonation structure generated by wedge-induced, oblique shocks in hydrogen-oxygennitrogen mixtures. Their simulations show a multidimensional detonation structure consisting of nonreactive oblique shock, induction zone, set of deflagration waves, and reactive shock (see Figure 15). The gaseous detonations in a channel with porous walls are investigated experimentally and numerically by Mazaheri et al. [121]. In this analysis both regular and irregular detonation waves were performed by Euler simulations. Wu and Lee [122] studied the stability of spinning detonation near the detonation limits. The detonation velocities as well as the wave structure are observed for long distance of propagation. They found that the fluctuation of local velocity, spinning detonation propagation, and fluctuations are increasing towards the limits. A two-dimensional structure of detonation wave in stoichiometric methane-air mixture in channel has been performed by Trotsyuk et al. [123]. From these calculations the irregular structure of detonation wave is obtained using a two-step kinetic model (see Figure 9).

\section{Reviews on Rotating Detonation Engines}

Voisekhovsky [124] performed experiments on continuously rotating detonations in 1960 . The fuel blending technique is proposed by George et al. [125] to achieve detonation initiation of hydrocarbon-air mixtures in a rotating detonation engine. Wu et al. observed that the stabilizing mechanism of hydrogen addition may be physical rather than chemical for fuel plenum. The sudden change of the stagnation pressure has an immediate influence on the average axial velocity at head end of the rotating detonation engine [126]. The rotating detonation combustion process and kinetic properties of reactive flow within the combustion chamber were studied by Meng et al. [127]. They observed the inner structure of the flow field of detonation at 1110 microseconds after ignition. The experimentation on two kinds of geometry of rotating detonation engine was studied by Kindracki et al. [128]. The thrust time profile as well as detonation velocity was calculated from pressure picks. Wang et al. [129] conducted experiments on continuous rotating detonation engine using hydrogen as a gaseous fuel. They observed that in annular combustor four different combustion patterns were observed as follows, that is, one wave in homorotating mode, two waves in homorotating mode, one couple in heterorotating mode, and two couples in heterorotating mode. Meng et al. [127] studied the three-dimensional numerical simulation investigating the combustion process and kinetic properties of reactive flow within the rotating detonation engine combustion chamber. Peng et al. [130] experimentally studied rotating detonation engine with the slot-orifice impinging injection method. They found that deflagration to detonation transition was observed in the annular combustion chamber and DDT time exhibited an obvious randomness and successful rate was up to $94 \%$.

\section{Some Results with CFD Analysis}

7.1. Nozzle Effect on Detonation Wave Acceleration. The performance of single phase pulse detonation engine was studied using computational fluid dynamics as shown in Figure 16. In these analyses detonation wave dynamic pressure and velocity contour were analyzed in pulse detonation engine with divergent nozzle. From the contour plots analysis it was observed that strong detonation wave dynamic pressure and velocity appeared in detonation tube [131].

7.2. Effect of Obstacle Geometry on Detonation Wave Temperature. The temperature contour plots of detonation wave in obstructed detonation tube having blockage ratio of $\mathrm{BR}=0.4$, $0.5,0.6$, and 0.7 at obstacle spacing of $S=4 \mathrm{~cm}$ are shown in Figure 17. The strong detonation wave propagation temperature of $3185 \mathrm{~K}$ was found in obstacle spacing of $S=4 \mathrm{~cm}$ and detonation wave acceleration temperature greatly depends on blockage ratio; the strong propagation temperature increases the thermodynamic performance, which was found in blockage ratio of $\mathrm{BR}=0.4[132]$.

7.3. Effect of Shchelkin Spiral on Detonation Wave Acceleration. The strong detonation wave dynamic pressure of $30.1 \times 10^{5} \mathrm{~Pa}$ was obtained in detonation tube with Shchelkin spiral configuration (see Figure 2), which is near about C-J detonation wave as shown in Figure 18(a). The detonation wave dynamic pressure of $10.1 \times 10^{5} \mathrm{~Pa}$ was obtained in clean configuration as shown in Figure 18(b). From the detonation wave magnitude, it was observed that Shchelkin spiral (see Figure 3) accelerates the detonation wave at same operating Mach number [133].

\section{Conclusions}

In the above comprehensive review study it is observed that there is more needed research in detonation wave flow path design and detonation wave acceleration at high supersonic Mach number. Due to this research drawback future possible research is that the change in exhaust nozzle design in the exit 


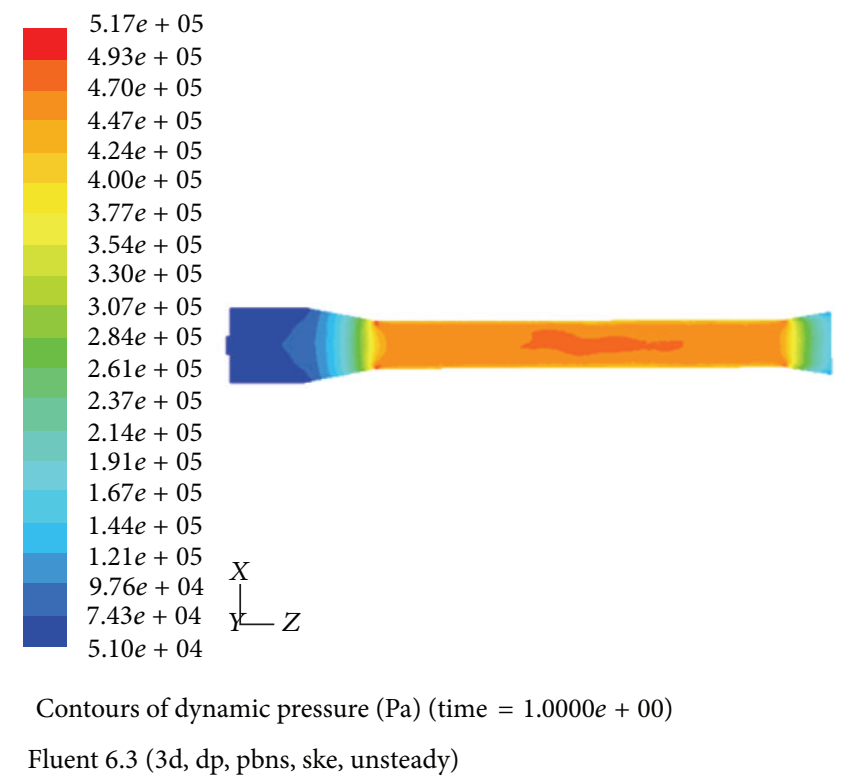

(a)

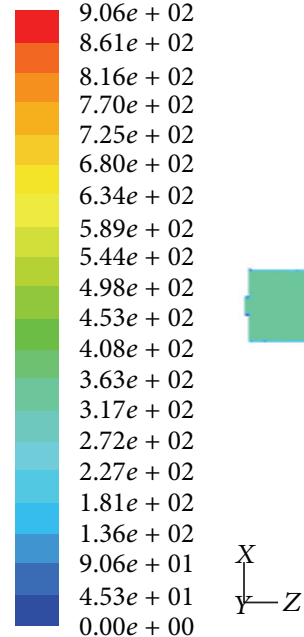

Contours of velocity magnitude $(\mathrm{m} / \mathrm{s})($ time $=1.0000 e+00)$

Fluent 6.3 (3d, dp, pbns, ske, unsteady)

(b)

FIGURE 16: Detonation wave (a) dynamic pressure and (b) velocity flow field contours analysis at $\mathrm{Ma}=1$ [131].

section of detonation tube can give better propulsion performance for detonation based pulse detonation engine. Further Shchelkin spiral improved the detonation wave initiation and acceleration inside the detonation tube of pulse detonation engine. Another successful detonation wave initiation as well as acceleration was observed inside the detonation tube with obstacles geometry. The U-bend shape detonation tube and ejectors at exit section of detonation tube can enhance deflagration flame to detonation wave transition. Exergetic efficiency of hydrogen-air detonation improves the thermodynamic performance of pulse detonation engine. The series of computational simulations performed the desired objectives of detonation combustion phenomena. Some further research scopes are as follows:

(a) The detonation combustion wave can be simulated using orifice plates and multiple reflections in detonation tube.

(b) Internal grooves effect on detonation wave characteristics can be analyzed.

(c) The combined effect of blockage and Shchelkin spiral can enhance the deflagration to detonation wave transition. 

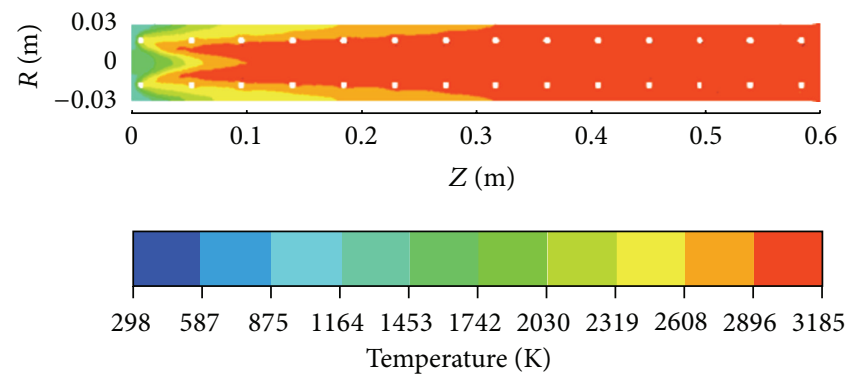

(a)
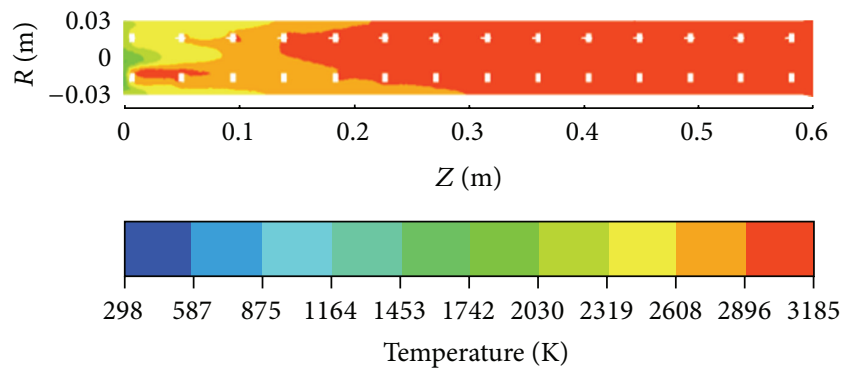

(c)
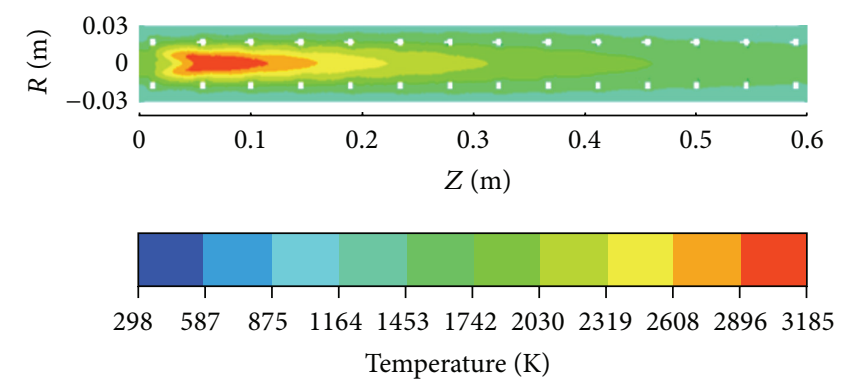

(b)
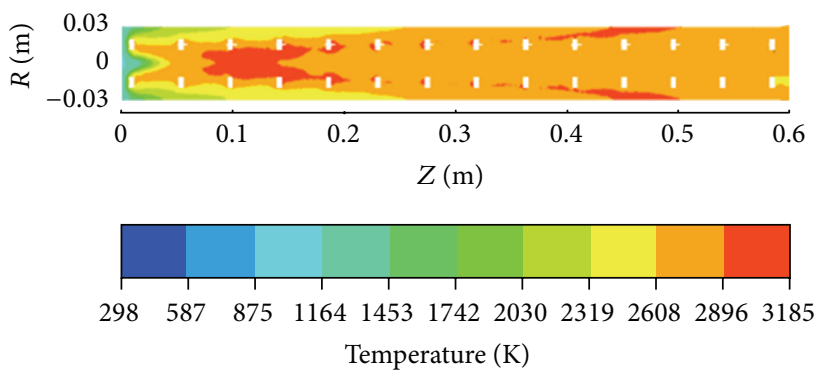

(d)

FIGURE 17: Contour of axial flame temperature distribution generated by an accelerating flame in obstacle landed detonation tube for (a) BR = 0.4 , (b) $\mathrm{BR}=0.5$, (c) $\mathrm{BR}=0.6$, and (d) $\mathrm{BR}=0.7$ at $\mathrm{Ma}=5$ for spacing of $S=4 \mathrm{~cm}$ [132].
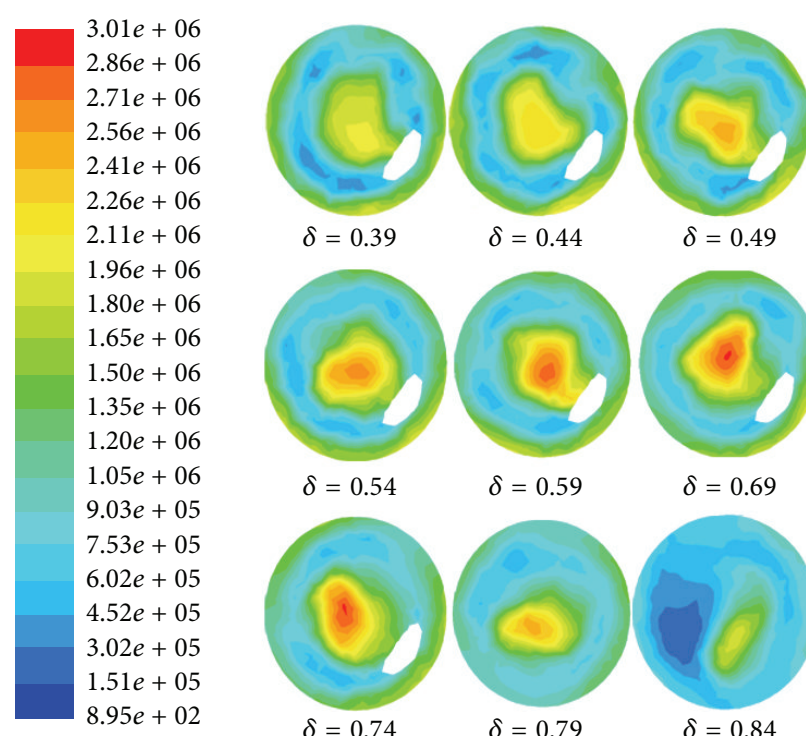

$\delta=0.39$

$\delta=0.44$

$\delta=0.49$
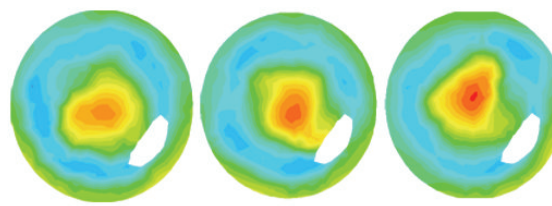

$\delta=0.54$

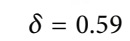

$\delta=0.69$

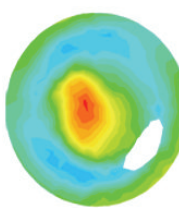

$\delta=0.74$

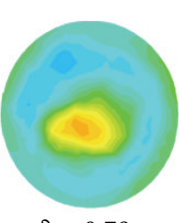

$\delta=0.79$

(a)

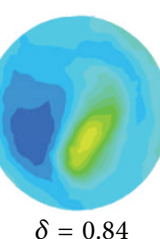

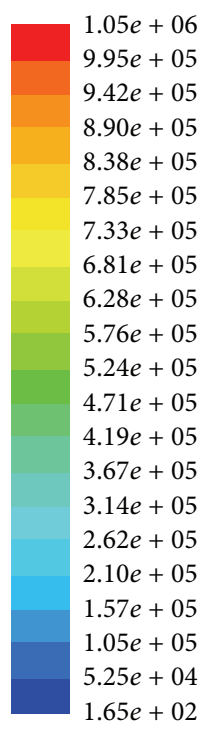

$65 e+02$

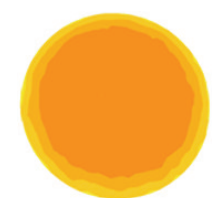

$\delta=0.39$

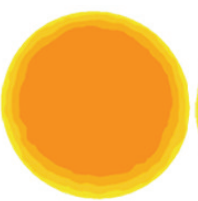

$\delta=0.54$

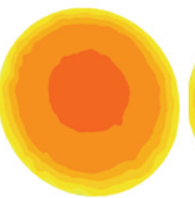

$\delta=0.74$

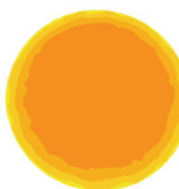

$\delta=0.44$

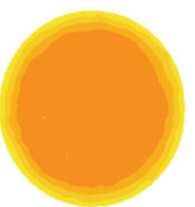

$\delta=0.59$

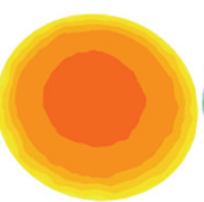

$\delta=0.79$

(b)

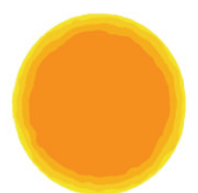

$\delta=0.49$

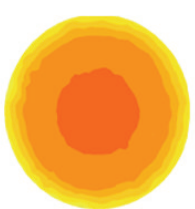

$\delta=0.69$

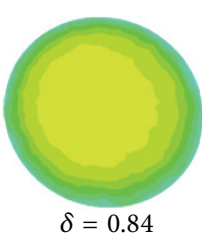

$\delta=0.84$

FIgURE 18: Contour plots evaluation of wave dynamic pressure (Pascal) field (a) in Shchelkin spiral and (b) without Shchelkin spiral in detonation tube at $\mathrm{Ma}=1.4[133]$.

(d) Detonation wave can be simulated using multi-injectors system.

(e) The detonation combustion can be simulated using different fuel utilization in pulse detonation engine combustor, like kerosene as a combustible fuel. (f) Heat transfer can be analyzed in detonation tube at different frequencies.

(g) Optimize the detonation parameter, that is, detonation wave pressure, temperature, density, and species mass fraction, by using different optimization 
technique to predict the propulsion performance of pulse detonation engine.

\section{Conflict of Interests}

The authors declare that there is no conflict of interests regarding the publication of this paper.

\section{References}

[1] G. D. Roy, S. M. Frolov, A. A. Borisov, and D. W. Netzer, "Pulse detonation propulsion: challenges, current status, and future perspective," Progress in Energy and Combustion Science, vol. 30, no. 6, pp. 545-672, 2004.

[2] Pratt and Whitney, "Pratt \& Whitney Acquires Propulsion technologyBusiness," 2003, http://www.pw.utc.com/pr_012301.asp.

[3] J. A. Nicholls, H. R. Wilkinson, and R. B. Morrison, "Intermittent detonation as a thrust-producing mechanism," Journal of Jet Propulsion, vol. 27, no. 5, pp. 534-541, 1956.

[4] L. Jin, W. Fan, K. Wang, and Z. Gao, "Review on the recent development of multi-mode combined detonation engine," International Journal of Turbo \& Jet Engines, vol. 30, no. 3, pp. 303-312, 2013.

[5] K. Kailasanath, "Review of propulsion applications of detonation waves," AIAA journal, vol. 38, no. 9, pp. 1698-1708, 2000.

[6] K. Kailasanath, "Recent developments in the research on pulse detonation engines," AIAA Journal, vol. 41, no. 2, pp. 145-159, 2003.

[7] R. D. Wilson and F. K. Lu, "Summary of recent research on detonation wave engines at UTA," in Proceedings of the International Workshop on Detonation for Propulsion, Busan, South Korea, November 2011.

[8] N. N. Smirnov, V. B. Betelin, V. F. Nikitin, Y. G. Phylippov, and J. Koo, "Detonation engine fed by acetylene-oxygen mixture," Acta Astronautica, vol. 104, no. 1, pp. 134-146, 2014.

[9] W. Chen, W. Fan, Q. Zhang, C. Peng, C. Yuan, and C. Yan, "Experimental investigation of nozzle effects on thrust and inlet pressure of an air-breathing pulse detonation engine," Chinese Journal of Aeronautics, vol. 25, no. 3, pp. 381-387, 2012.

[10] Q. Li, W. Fan, C.-J. Yan, C.-Q. Hu, and B. Ye, "Experimental investigation on performance of pulse detonation rocket engine model," Chinese Journal of Aeronautics, vol. 20, no. 1, pp. 9-14, 2007.

[11] Y. Yan, W. Fan, K. Wang, and Y. Mu, "Experimental investigation of the effect of bell-shaped nozzles on the two-phase pulse detonation rocket engine performance," Combustion, Explosion and Shock Waves, vol. 47, no. 3, pp. 335-342, 2011.

[12] D. Allgood, E. Gutmark, J. Hoke, R. Bradley, and F. Schauer, "Performance measurements of multicycle pulse-detonationengine exhaust nozzles," Journal of Propulsion and Power, vol. 22, no. 1, pp. 70-77, 2006.

[13] C. Peng, W. Fan, L. Zheng, Z. Wang, and C. Yuan, "Experimental investigation on valveless air-breathing dual-tube pulse detonation engines," Applied Thermal Engineering, vol. 51, no. 1-2, pp. 1116-1123, 2013.

[14] Y. Yan, W. Fan, K. Wang, X.-D. Zhu, and Y. Mu, "Experimental investigations on pulse detonation rocket engine with various injectors and nozzles," Acta Astronautica, vol. 69, no. 1-2, pp. 3947, 2011.

[15] J. Kasahara, K. Marsuoka, T. Nakamichi, M. Esumi, A. Matsuo, and I. Funaki, "Study of high frequency rotary valve pulse detonation rocket engine," in Proceedings of the DWP Workshop, pp. 11-13, Bourges, France, 2011.

[16] M. Cooper, S. Jackson, J. Austin, E. Wintenberger, and J. E. Shepherd, "Direct experimental impulse measurements for detonations and deflagrations," in Proceedings of the 37th Joint Propulsion Conference and Exhibit, AIAA-2001-3812, Salt Lake City, Utah, USA, 2001.

[17] J. B. Hinkey, J. T. Williams, S. E. Henderson, and T. R. A. Bussing, "Rotary-valved, multiple-cycle, pulse detonation engine experimental demonstration," in Proceedings of the 33rd Joint Propulsion Conference and Exhibit, AIAA, pp. 27-46, Seattle, Wash, USA, 1997.

[18] J. Kasahara, A. Hasegawa, T. Nemoto, H. Yamaguchi, T. Yajima, and T. Kojima, "Performance validation of a single-tube pulse detonation rocket system," Journal of Propulsion and Power, vol. 25, no. 1, pp. 173-180, 2009.

[19] J.-L. Li, W. Fan, C.-J. Yan, H.-Y. Tu, and K.-C. Xie, "Performance enhancement of a pulse detonation rocket engine," Proceedings of the Combustion Institute, vol. 33, no. 2, pp. 2243-2254, 2011.

[20] K. Asato, T. Miyasaka, Y. Watanabe, and K. Tanabashi, "Combined effects of vortex flow and the Shchelkin spiral dimensions on characteristics of deflagration-to-detonation transition," Shock Waves, vol. 23, no. 4, pp. 325-335, 2013.

[21] T. H. New, P. K. Panicker, F. K. Lu, and H. M. Tsai, "Experimental investigations on DDT enhancements by shchelkin spirals in a PDE," AIAA 2006-552, AIAA, 2006.

[22] W. Wang, H. Qiu, W. Fan, and C. Xiong, "Experimental study on DDT characteristics in spiral configuration pulse detonation engines," International Journal of Turbo \& Jet Engines, vol. 30, no. 3, pp. 261-270, 2013.

[23] P. K. Panicker, F. K. Lu, and D. R. Wilson, "Practical methods for reducing the deflagration-to-detonation transition length for pulse detonation engines," in Proceedings of the 9th International Symposium on Experimental and Computational Aerothermodynamics of Internal Flows (ISAIF '09), Gyeongju, Republic of Korea, September 2009.

[24] D. Valiev, V. Bychkov, V. Akkerman, C. K. Law, and L.-E. Eriksson, "Flame acceleration in channels with obstacles in the deflagration-to-detonation transition," Combustion and Flame, vol. 157, no. 5, pp. 1012-1021, 2010.

[25] A. V. Gaathaug, K. Vaagsaether, and D. Bjerketvedt, "Experimental and numerical investigation of DDT in hydrogen-air behind a single obstacle," International Journal of Hydrogen Energy, vol. 37, no. 22, pp. 17606-17615, 2012.

[26] I. O. Moen, M. Donato, R. Knystautas, and J. H. Lee, "Flame acceleration due to turbulence produced by obstacles," Combustion and Flame, vol. 39, no. 1, pp. 21-32, 1980.

[27] T. Ogawa, V. N. Gamezo, and E. S. Oran, "Flame acceleration and transition to detonation in an array of square obstacles," Journal of Loss Prevention in the Process Industries, vol. 26, no. 2, pp. 355-362, 2013.

[28] C. Johansen and G. Ciccarelli, "Numerical simulations of the flow field ahead of an accelerating flame in an obstructed channel," Combustion Theory and Modelling, vol. 14, no. 2, pp. 235-255, 2010.

[29] V. N. Gamezo, T. Ogawa, and E. S. Oran, "Flame acceleration and DDT in channels with obstacles: effect of obstacle spacing," Combustion and Flame, vol. 155, no. 1-2, pp. 302-315, 2008.

[30] C. Johansen and G. Ciccarelli, "Numerical simulations of the flow field ahead of an accelerating flame in an obstructed channel," Combustion Theory and Modelling, vol. 14, no. 2, pp. 235-255, 2010. 
[31] D. A. Kessler, V. N. Gamezo, E. S. Oran, and R. K. Zipf, "Simulation of deflagration-to-detonation transition in premixed $\mathrm{CH}_{4}$ air in large-scale channels with obstacles," in Proceedings of the 47th AIAA Aerospace Sciences Meeting Including the New Horizons Forum and Aerospace Exposition, Orlando, Fla, USA, January 2009.

[32] Y. Huang, H. Tang, J. Li, and C. Zhang, "Studies of DDT enhancement approaches for kerosene-fueled small-scale pulse detonation engines applications," Shock Waves, vol. 22, no. 6, pp. 615-625, 2012.

[33] W. Rudy, R. Porowski, and A. Teodorczyk, "Propagation of hydrogen-air detonation in tube with obstacles," Journal of Power Technologies, vol. 91, no. 3, pp. 122-129, 2011.

[34] D. I. Baklanov, S. V. Golovastov, V. V. Golub, N. V. Semin, and V. V. Valodin, "Model of low-thrust pulse detonation device with valve less fuel feed," Progress in Propulsion Physics, vol. 1, pp. 341352, 2009.

[35] D. E. Paxson, "Performance impact of deflagration to detonation transition enhancing obstacles," in Proceedings of the 47th Aerospace Sciences Meeting, AIAA-2009-502, AIAA, Orlando, Fla, USA, January 2009.

[36] S. M. Frolov, "Fast deflagration-to-detonation transition," Russian Journal of Physical Chemistry B, vol. 2, no. 3, pp. 442-455, 2008, Pleiades Publishing, 2008, Original Russian Text, S. M. Frolov, 2008, Published in Khimicheskaya Fizika, vol. 27, no. 6, pp. 32-46, 2008.

[37] A. Teodorczyk, "Scale effects on hydrogen-air fast deflagrations and detonations in small obstructed channels," Journal of Loss Prevention in the Process Industries, vol. 21, no. 2, pp. 147-153, 2008.

[38] S. M. Frolov, V. S. Aksenov, and I. O. Shamshin, "Detonation propagation through U-bends," in Nonequilibrium Processes. Combustion and detonation, G. Roy, S. Frolov, and A. Starik, Eds., vol. 1, pp. 348-364, Torus Press, Moscow, Russia, 2005.

[39] S. M. Frolov, V. S. Aksenov, and I. O. Shamshin, "Reactive shock and detonation propagation in U-bend tubes," Journal of Loss Prevention in the Process Industries, vol. 20, no. 4-6, pp. 501508, 2007.

[40] C. Tharratt, "The propulsive duct," Aircraft Engineering and Aerospace Technology, vol. 37, no. 12, pp. 359-371, 1965.

[41] F. Zheng, A. V. Kuznetsov, W. L. Roberts, and D. E. Paxson, "Influence of geometry on starting vortex and ejector performance," Journal of Fluids Engineering, vol. 133, no. 5, Article ID 051204, 2011.

[42] G. Ciccarelli, C. T. Johansen, and M. Parravani, "The role of shock-flame interactions on flame acceleration in an obstacle laden channel," Combustion and Flame, vol. 157, no. 11, pp. 21252136, 2010.

[43] V. N. Gamezo, T. Ogawa, and E. S. Oran, "Numerical simulations of flame propagation and DDT in obstructed channels filled with hydrogen-air mixture," Proceedings of the Combustion Institute, vol. 31, no. 2, pp. 2463-2471, 2007.

[44] C. T. Johansen and G. Ciccarelli, "Visualization of the unburned gas flow field ahead of an accelerating flame in an obstructed square channel," Combustion and Flame, vol. 156, no. 2, pp. 405416, 2009.

[45] S. M. Frolo, V. S. Aksenov, and I. O. Shamshin, "Shock wave and detonation propagation through U-bend tubes," Proceedings of the Combustion Institute, vol. 31, no. 2, pp. 2421-2428, 2007.

[46] I. V. Semenov, P. S. Utkin, V. V. Markov, S. M. Frolov, and V. S. Aksenov, "Numerical and experimental investigation of detonation initiation in profiled tubes," Combustion Science and Technology, vol. 182, no. 11-12, pp. 1735-1746, 2010.

[47] D. Allgood and E. Gutmark, "Experimental investigation of a pulse detonation engine with a $2 \mathrm{D}$ ejector," in Proceedings of the 42nd AIAA Aerospace Sciences Meeting and Exhibit, AIAA2004-864, Reno, Nev, USA, January 2004.

[48] Y. Yan, W. Fan, and Y. Mu, "Preliminary experimental investigation on detonation initiation in the ejector of a pulse detonation rocket engine," International Journal of Turbo \& Jet Engines, vol. 29, no. 4, pp. 299-307, 2012.

[49] Q. D. Bai and C. S. Weng, "Experimental study of ejectors' effect on the performance of pulse detonation rocket engine," Applied Mechanics and Materials, vol. 628, pp. 293-298, 2014.

[50] G. Canteins, F. Franzetti, E. Zocłońska, B. A. Khasainov, R. Zitoun, and D. Desbordes, "Experimental and numerical investigations on PDE performance augmentation by means of an ejector," Shock Waves, vol. 15, no. 2, pp. 103-112, 2006.

[51] X. Cha, Y. Chuan-Jun, and Q. Hua, "Analysis of an air-breathing pulsed detonation engine with bypass and ejector," International Journal of Turbo \& Jet-Engines, vol. 25, no. 2, pp. 129-136, 2008.

[52] R. J. Santoro, P. Sibtosh, R. Shehadeh, S. Saretto, and S. Y. Lee, "Experimental study of a pulse detonation engine driven ejector," in Proceedings of the 39th AIAA/ASME/SAE/ASEE Joint Propulsion Conference and Exhibit, AIAA 2003-4972, Huntsville, Ala, USA, July 2003.

[53] J. L. Hoke, A. G. Naples, L. P. Goss, and F. R. Schauer, "Schlieren imaging of a single-ejector multi-tube pulsed detonation engine," in Proceedings of the 47th AIAA Aerospace Sciences Meeting Including The New Horizons Forum and Aerospace Exposition, Orlando, Fla, USA, January 2009.

[54] J. Wilson, A. Sgondea, D. E. Paxson, and B. N. Rosenthal, "Parametric investigation of thrust augmentation by ejectors on a pulsed detonation tube," Journal of Propulsion and Power, vol. 23, no. 1, pp. 108-115, 2007.

[55] D. E. Paxson, P. J. Litke, F. R. Schaue, R. P. Bradley, and J. L. Hoke, "Performance assessment of a large scale pulsejet-driven ejector system," in Proceedings of the 44th AIAA Aerospace Sciences Meeting and Exhibit, Reno, Nev, USA, January 2006.

[56] A. J. Glaser, N. Caldwell, E. Gutmark, J. Hoke, R. Bradley, and F. Schauer, "Study on the operation of pulse-detonation enginedriven ejectors," Journal of Propulsion and Power, vol. 24, no. 6, pp. 1324-1331, 2008.

[57] P. Changxin, F. Wei, Z. Qun, Y. Cheng, C. Wenjuan, and Y. Chuanjun, "Experimental study of an air-breathing pulse detonation engine ejector," Experimental Thermal and Fluid Science, vol. 35, no. 6, pp. 971-977, 2011.

[58] A. E. Korobov and S. V. Golovastov, "Numerical investigation of the ejector influence on the effectiveness of a jet nozzle of a detonation engine," High Temperature, vol. 53, no. 1, pp. 118-123, 2015.

[59] X. Huang, M. Li, Y. Yuan, Y. Xiong, and L. Zheng, "Experimental investigation on noise radiation characteristics of pulse detonation engine-driven ejector," Advances in Mechanical Engineering, vol. 7, no. 6, 2015.

[60] H. Qiu, C. Xiong, C.-J. Yan, and L.-X. Zheng, "Effect of aerodynamic valve on backflow in pulsed detonation tube," Aerospace Science and Technology, vol. 25, no. 1, pp. 1-15, 2013.

[61] K. Matsuoka, M. Esumi, K. B. Ikeguchi, J. Kasahara, A. Matsuo, and I. Funaki, "Optical and thrust measurement of a pulse detonation combustor with a coaxial rotary valve," Combustion and Flame, vol. 159, no. 3, pp. 1321-1338, 2012. 
[62] K. Matsuoka, T. Mukai, and T. Endo, "Development of a liquidpurge method for high-frequency operation of pulse detonation combustor," Combustion Science and Technology, vol. 187, no. 5, pp. 747-764, 2015.

[63] W. Fan, C.-J. Yan, Q. Li, Y.-Q. Ding, and J.-H. Li, “Experimental investigations on detonability limits of pulse detonation rocket engine," International Journal of Turbo \& Jet Engines, vol. 23, no. 4, pp. 277-282, 2006.

[64] S. M. Frolov, V. S. Aksenov, and V. S. Ivanov, "Experimental demonstration of the operation process of a pulse-detonation liquid rocket engine," Russian Journal of Physical Chemistry B, vol. 5, no. 4, pp. 664-667, 2011.

[65] V. E. Tangirala, A. Rasheed, and A. J. Dean, "Performance of a pulse detonation combustor-based hybrid engine," in Proceedings of the ASME Turbo Expo: Power for Land, Sea and Air, vol. 3 of ASME Proceedings, Paper no. GT2007-28056, pp. 403-414, Montreal, Canada, May 2007.

[66] J. Li, W. Fan, C. Yan, and Q. Li, "Experimental investigations on detonation initiation in a kerosene-oxygen pulse detonation rocket engine," Combustion Science and Technology, vol. 181, no. 3, pp. 417-432, 2009.

[67] C. A. Stevens, P. I. King, F. R. Schauer, and J. L. Hoke, "An experimental study of detonation diffraction and shock initiation," in Proceedings of the 51st AIAA Aerospace Sciences Meeting Including the New Horizons Forum and Aerospace Exposition, AIAA 2013-1027, Grapevine, Tex, USA, January 2013.

[68] W. Fan, C. Yan, X. Huang, Q. Zhang, and L. Zheng, "Experimental investigation on two-phase pulse detonation engine," Combustion and Flame, vol. 133, no. 4, pp. 441-450, 2003.

[69] N. Mehrjoo, R. Portaro, and H. D. Ng, "A technique for promoting detonation transmission from a confined tube into larger area for pulse detonation engine applications," Propulsion and Power Research, vol. 3, no. 1, pp. 9-14, 2014.

[70] X. Huang, Y. Yan, Y. Mu, L. Zheng, and L. Chen, "Experimental investigation on heating kerosene using thrust tube waste heat of pulse detonation engine," International Journal of Turbo \& Jet-Engines, vol. 30, no. 4, pp. 339-345, 2013.

[71] J. Deng, L. Zheng, C. Yan, L. Jiang, C. Xiong, and N. Li, "Experimental investigation of a puls detonation combustor integrated with a turbine," International Journal of Turbo \& JetEngines, vol. 27, no. 2, pp. 119-134, 2010.

[72] X. Cha, Y. Chuan-Jun, and Q. Hua, "Experiments and numerical simulation of exhaust temperature of pulsed detonation engine," International Journal of Turbo and Jet Engines, vol. 25, no. 2, pp. 137-144, 2008.

[73] T. Morozumi, K. Matsuoka, R. Sakamota, Y. Fujiwara, and J. Kasahara, "Study on a four-cylinder pulse detonation rocket engine with a coaxial high frequency rotary valve," in Proceedings of the 51st AIAA Aerospace Sciences Meeting including the New Horizones Forum and Aerospace Exposition, AIAA 2013027, Dallas, Tex, USA, January 2013.

[74] R. Zitoun and D. Desbordes, "Propulsive performances of pulsed detonations," Combustion Science and Technology, vol. 144, no. 1, pp. 93-114, 1999.

[75] Z.-C. Fan, W. Fan, H.-Y. Tu, J.-L. Li, and C.-J. Yan, "The effect of fuel pretreatment on performance of pulse detonation rocket engines," Experimental Thermal and Fluid Science, vol. 41, pp. 130-142, 2012.

[76] S. K. Soni, A. Singh, M. Sandhu, A. Goel, and R. K. Sharma, "Numerical simulation to investigate the effect of obstacle on detonation wave propagation in a pulse detonation engine combustor," International Journal of Emerging Technology and Advanced Engineering, vol. 3, no. 3, pp. 458-464, 2013.

[77] M. R. Amin, H. Z. Rouf, and J.-L. Cambier, "Numerical investigation on the effects of nozzle geometry on the performance of a pulse detonation engine," Journal of Mechanical Engineering, vol. 51, no. 7-8, pp. 484-490, 2005.

[78] V. N. Tangirala, A. J. Dean, N. Tsuboi, and A. K. Hayashi, "Performance of a pulse detonation engine under subsonic and supersonic flight conditions," in Proceedings of the 45th AIAA Aerospace Sciences Meeting and Exhibit, AIAA 2007-1245, Reno, Nev, USA, January 2007.

[79] Y. Shao, M. Liu, and J. Wang, "Continuous detonation engine and effects of different types of nozzle on its propulsion performance," Chinese Journal of Aeronautics, vol. 23, no. 6, pp. 647-652, 2010.

[80] F. Ma, J.-Y. Choi, and V. Yang, “Thrust chamber dynamics and propulsive performance of multitude pulse detonation engines," Journal of Propulsion and Power, vol. 21, no. 4, pp. 681-691, 2005.

[81] E. Wintenberger and J. E. Shepherd, "Model for the performance of airbreathing pulse-detonation engines," Journal of Propulsion and Power, vol. 22, no. 3, pp. 593-603, 2006.

[82] K. Kailasanath and G. Patnaik, "Performance estimates of pulsed detonation engines," Proceedings of the Combustion Institute, vol. 28, no. 1, pp. 595-601, 2000.

[83] F. Ma, J.-Y. Choi, and V. Yang, "Internal flow dynamics in a valveless airbreathing pulse detonation engine," Journal of Propulsion and Power, vol. 24, no. 3, pp. 479-490, 2008.

[84] S. M. Frolov and V. S. Aksenov, "Deflagration-to-detonation transition in a kerosene-air mixture," Doklady Physical Chemistry, vol. 416, no. 1, pp. 261-264, 2007.

[85] C. Johansen and G. Ciccarelli, "Modeling the initial flame acceleration in an obstructed channel using large eddy simulation," Journal of Loss Prevention in the Process Industries, vol. 26, no. 4, pp. 571-585, 2013.

[86] S. M. Frolov, V. S. Aksenov, and I. O. Shamshin, "Propagation of shock and detonation waves in channels with U-shaped bends of limiting curvature," Russian Journal of Physical Chemistry B, vol. 2, no. 5, pp. 759-774, 2008.

[87] S. Otsuka, M. Suzuki, and M. Yamamoto, "Numerical investigation on detonation wave through U-bend," Journal of Thermal Science, vol. 19, no. 6, pp. 540-544, 2010.

[88] F. Zheng, A. V. Kuznetsov, W. L. Roberts, and D. E. Paxson, "Numerical study of a pulsejet-driven ejector," in Proceedings of the 45th AIAA/ASME/SAE/ASEE Joint Propulsion Conference \& Exhibit, Denver, Colorado, August 2009.

[89] D. Allgood, E. Gutmark, J. Hoke, R. Bradley, and F. Schauer, "Performance studies of pulse detonation engine ejectors," Journal of Propulsion and Power, vol. 24, no. 6, pp. 1317-1323, 2008.

[90] H.-H. Zhang, Z.-H. Chen, X.-H. Sun, X.-H. Jiang, and B.M. Li, "Numerical investigations on the thrust augmentation mechanisms of ejectors driven by pulse detonation engines," Combustion Science and Technology, vol. 183, no. 10, pp. 10691082, 2011.

[91] M. R. Nalim, Z. A. Izzy, and P. Akbari, "Rotary wave-ejector enhanced pulse detonation engine," Shock Waves, vol. 22, no. 1, pp. 23-38, 2012.

[92] T. H. Yi, D. R. Wilson, and F. K. Lu, "Detonation wave propagation in an ejector augmented pulse detonation rocket," in Proceedings of the 44th AIAA Aerospace Sciences Meeting and Exhibit, Reno, Nev, USA, January 2006. 
[93] W. A. Stoddard, M. Mihaescu, and E. Gutmark, "Simulation of detonation based cycles in tubes with multiple openings," in Proceedings of the 46th AIAA/ASME/SAE/ASEE Joint Propulsion Conference \& Exhibit, AIAA 2010-6745, Nashville,Tenn, USA, July 2010.

[94] X.-M. Tan, J.-Z. Zhang, and X.-T. Wang, "Effects of pulse detonation wave on film dynamics," Engineering Applications of Computational Fluid Mechanics, vol. 5, no. 4, pp. 499-505, 2011.

[95] X. He and A. R. Karagozian, "Numerical simulation of pulse detonation engine phenomena," Journal of Scientific Computing, vol. 19, no. 1-3, pp. 201-224, 2003.

[96] S. Yungster, K. Radhakrishnan, and K. Breisacher, "Computational study of $\mathrm{NO}_{x}$ formation in hydrogen-fuelled pulsed detonation engines," Combustion Theory and Modelling, vol. 10, no. 6, pp. 981-1002, 2013.

[97] H. Taniguchi, K. Mouri, T. Nakahara, and N. Arai, "Exergy analysis on combustion and energy conversion processes," Energy, vol. 30, no. 2-4, pp. 111-117, 2005.

[98] R. Petela, "Application of exergy analysis to the hydrodynamic theory of detonation in gases," Fuel Processing Technology, vol. 67, no. 2, pp. 131-145, 2000.

[99] A. Rouboa, V. Silva, and N. Couto, "Exergy analysis in hydrogen-air detonation," Journal of Applied Mathematics, vol. 2012, Article ID 502979, 16 pages, 2012.

[100] R. Bellini and F. K. Lu, "Exergy analysis of a pulse detonation power device," in Proceedings of the 10th Brazilian Congress of Thermal Sciences and Engineering (ENCIT '04), Brazilian Society of Mechanical Sciences and Engineering, ABCM, Rio de Janeiro, Brazil, November 2004.

[101] T. E. Hutchins and M. Metghalchi, "Energy and exergy analyses of the pulse detonation engine," Journal of Engineering for Gas Turbines and Power, vol. 125, no. 4, pp. 1075-1080, 2003.

[102] R. Bellini and F. K. Lu, "Exergy analysis of a pulse detonation power device," Journal of Propulsion and Power, vol. 26, no. 4, pp. 875-877, 2010.

[103] M. Safari, M. R. H. Sheikhi, M. Janbozorgi, and H. Metghalchi, "Entropy transport equation in large eddy simulation for exergy analysis of turbulent combustion systems," Entropy, vol. 12, no. 3, pp. 434-444, 2010.

[104] Z. Wu, S. Zhou, and L. An, "The second law (exergy) analysis of hydrogen," Journal of Sustainable Development, vol. 4, no. 1, pp. 260-263, 2011.

[105] W. Hu, Z. Deng, and G. Xie, "Energy loss in pulse detonation engine due to fuel viscosity," Mathematical Problems in Engineering, vol. 2014, Article ID 735926, 5 pages, 2014.

[106] S. K. Som and N. Y. Sharma, "Energy and exergy balance in the process of spray combustion in a gas turbine combustor," Journal of Heat Transfer, vol. 124, no. 5, pp. 828-836, 2002.

[107] T. Endo and T. Fujiwara, "A simplified analysis on a pulse detonation engine model," Transactions of the Japan Society for Aeronautical and Space Sciences, vol. 44, no. 146, pp. 217-222, 2002.

[108] Y. B. Zel'dovich, "To the question of energy use of detonation combustion," Journal of Propulsion and Power, vol. 22, no. 3, pp. 588-592, 2006.

[109] W. H. Heiser and D. T. Pratt, "Thermodynamic cycle analysis of pulse detonation engines," Journal of Propulsion and Power, vol. 18, no. 1, pp. 68-76, 2002.

[110] Y. Wu, F. Ma, and V. Yang, "System performance and thermodynamic cycle analysis of airbreathing pulse detonation engines," Journal of Propulsion and Power, vol. 19, no. 4, pp. 556-567, 2003.
[111] C. Li and K. Kailasanath, "Partial fuel filling in pulse detonation engines," Journal of Propulsion and Power, vol. 19, no. 5, pp. 908916, 2003.

[112] M. Cooper, J. E. Shepherd, and F. Schauer, "Impulse correlation for partially filled detonation tubes," Journal of Propulsion and Power, vol. 20, no. 5, pp. 947-950, 2004.

[113] F. Schauer, J. Stutrud, and R. Bradly, "Detonation initiation studies and performance results for pulsed detonation engine applications," in Proceedings of the 39th AIAA Aerospace Sciences Meeting, AIAA 2001-1129, Reno, Nev, USA, January 2001.

[114] K. Kim, G. Varsi, and L. H. Back, "Blast wave analysis for detonation propulsion," AIAA Journal, vol. 15, no. 10, pp. 1500 $1502,1977$.

[115] F. A. Bykovskii and E. F. Vedernikov, "Continuous detonation of a subsonic flow of a propellant," Combustion, Explosion and Shock Waves, vol. 39, no. 3, pp. 323-334, 2003.

[116] N. Smirnov, "Pulse detonation engines: advantages and limitations," in Advanced Combustion and Aerothermal Technologies, NATO Science for Peace and Security Series C: Environmental Security, pp. 353-363, Springer, Dordrecht, The Netherlands, 2007.

[117] M. Z. Ahmad Faiz, M. A. Wahid, K. M. Saqr, and U. Haffis, "Single pulse detonation study of natural gas," in Proceedings of the International Conference on Theoretical and Applied Mechanics, and International Conference on Fluid Mechanics and Heat \& Mass Transfer (MECHANICS-HEAPFL '10), pp. 7883, Stevens Point, Wis, USA, 2010.

[118] M. I. Radulescu and R. K. Hanson, "Effect of heat loss on pulse-detonation-engine flow fields and performance," Journal of Propulsion and Power, vol. 21, no. 2, pp. 274-285, 2005.

[119] R. Driscoll, W. Stoddard, A. St George, and E. Gutmark, "Shock transfer and shock-initiated detonation in a dual pulse detonation engine/crossover system," AIAA Journal, vol. 53, no. 1, pp. 132-139, 2015.

[120] C. Li, K. Kailasanath, and E. S. Oran, "Detonation structures behind oblique shocks," Physics of Fluids, vol. 6, no. 4, pp. 16001611, 1994.

[121] K. Mazaheri, Y. Mahmoudi, M. Sabzpooshani, and M. I. Radulescu, "Experimental and numerical investigation of propagation mechanism of gaseous detonations in channels with porous walls," Combustion and Flame, vol. 162, no. 6, pp. 26382659, 2015.

[122] Y. Wu and H. S. J. Lee, "Stability of spinning detonation waves," Combustion and Flame, vol. 162, no. 6, pp. 2660-2669, 2015.

[123] A. V. Trotsyuk, P. A. Fomin, and A. A. Vasil'ev, "Numerical study of cellular detonation structures of methane mixtures," Journal of Loss Prevention in the Process Industries, vol. 36, pp. 394-403, 2015.

[124] B. V. Voisekhovsky, "A spin stationary detonation," Applied Mechanics and Technical Physics, vol. 3, pp. 157-164, 1960.

[125] A. George, R. Driscoll, V. Anand, D. Munday, and E. J. Gutmark, "Fuel blending as a means to achieve initiation in a rotating detonation engine," in Proceedings of the 53rd AIAA Aerospace Sciences Meeting, AIAA SciTech, Kissimmee, Fla, USA, January 2015.

[126] D. Wu, Y. Liu, Y. Liu, and J. Wang, "Numerical investigations of the restabilization of hydrogen-air rotating detonation engines," International Journal of Hydrogen Energy, vol. 39, no. 28, pp. 15803-15809, 2014.

[127] L. Meng, Z. Shuang, W. Jianping, and C. Yifeng, "Parallel three-dimensional numerical simulation of rotating detonation 
engine on graphics processing units," Computers \& Fluids, vol. 110, pp. 36-42, 2015.

[128] J. Kindracki, P. Wolański, and Z. Gut, "Experimental research on the rotating detonation in gaseous fuels-oxygen mixtures," Shock Waves, vol. 21, no. 2, pp. 75-84, 2011.

[129] C. Wang, W. Liu, S. Liu, L. Jiang, and Z. Lin, "Experimental investigation on detonation combustion patterns of hydrogen/ vitiated air within annular combustor," Experimental Thermal and Fluid Science, vol. 66, pp. 269-278, 2015.

[130] L. Peng, D. Wang, X. Wu, H. Ma, and C. Yang, "Ignition experiment with automotive spark on rotating detonation engine," International Journal of Hydrogen Energy, vol. 40, no. 26, pp. 8465-8474, 2015.

[131] P. Debnath and K. M. Pandey, "Performance investigation on single phase pulse detonation engine using computational fluid dynamics," in Proceedings of the ASME International Mechanical Engineering Congress \& Exposition (IMECE '13), IMECE201366274, San Diego, Calif, USA, November 2013.

[132] P. Debnath and K. M. Pandey, "Effect of blockage ratio on detonation flame acceleration in pulse detonation combustor using CFD," Applied Mechanics and Materials, vol. 656, pp. 6471, 2014.

[133] P. Debnath and K. M. Pandey, "Computational study of deflagration to detonation transition in pulse detonation engine using shchelkin spiral," Applied Mechanics and Materials, vol. 772, pp. 136-140, 2015. 


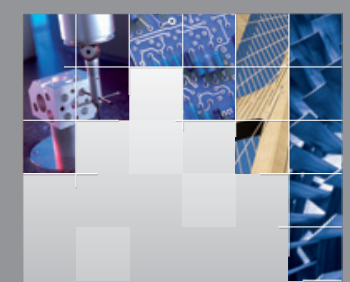

\section{Enfincering}
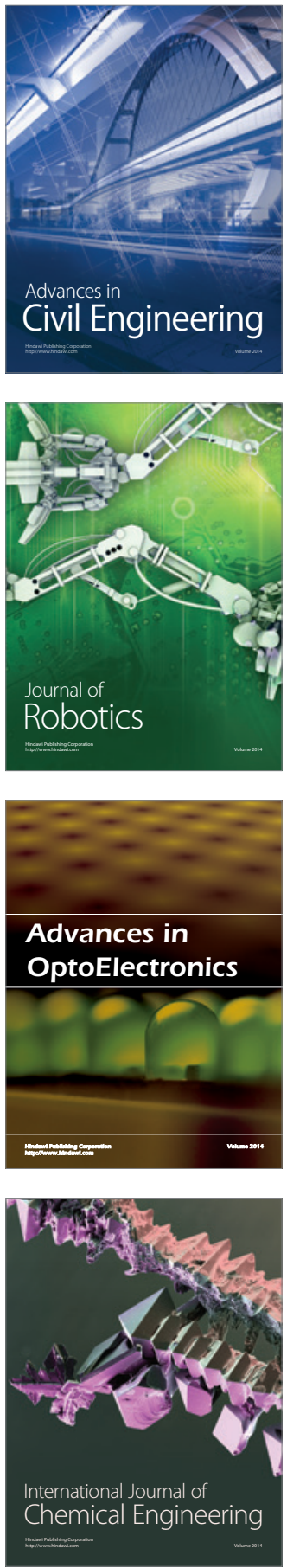

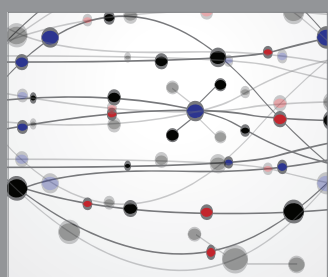

The Scientific World Journal

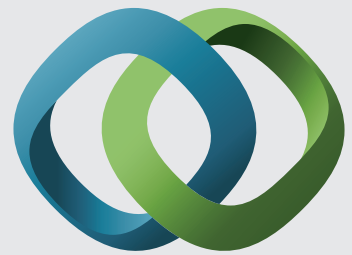

\section{Hindawi}

Submit your manuscripts at

http://www.hindawi.com
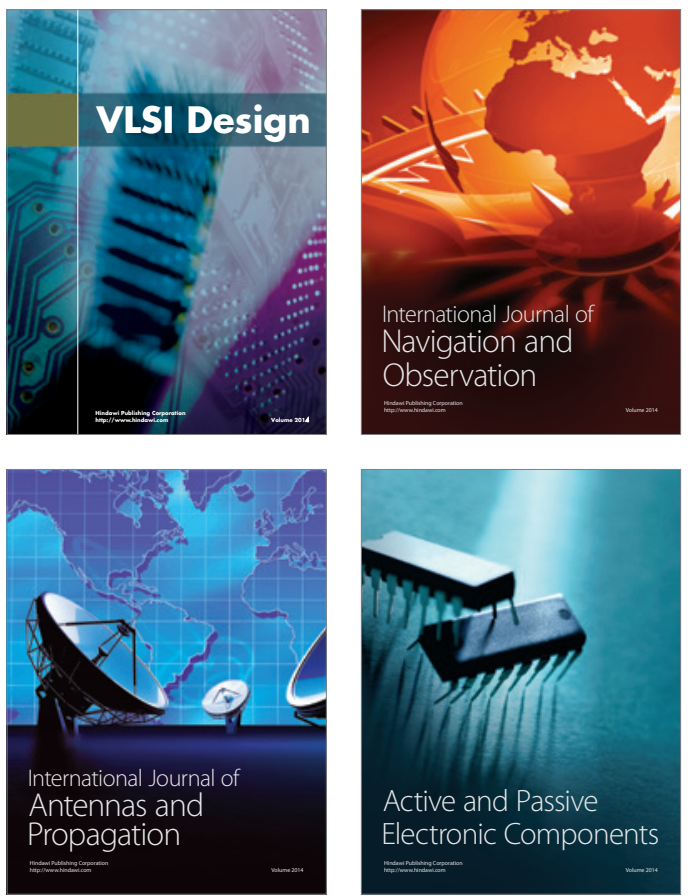
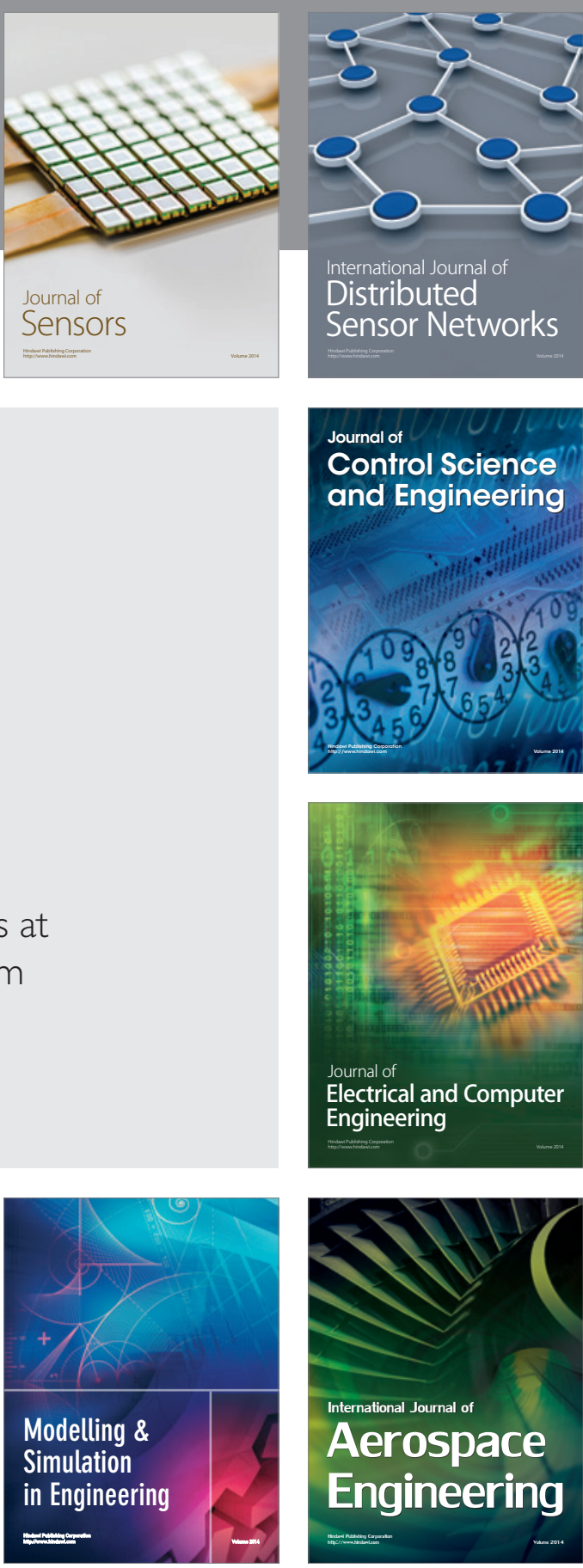

International Journal of

Distributed

Sensor Networks

Journal of

Control Science

and Engineering
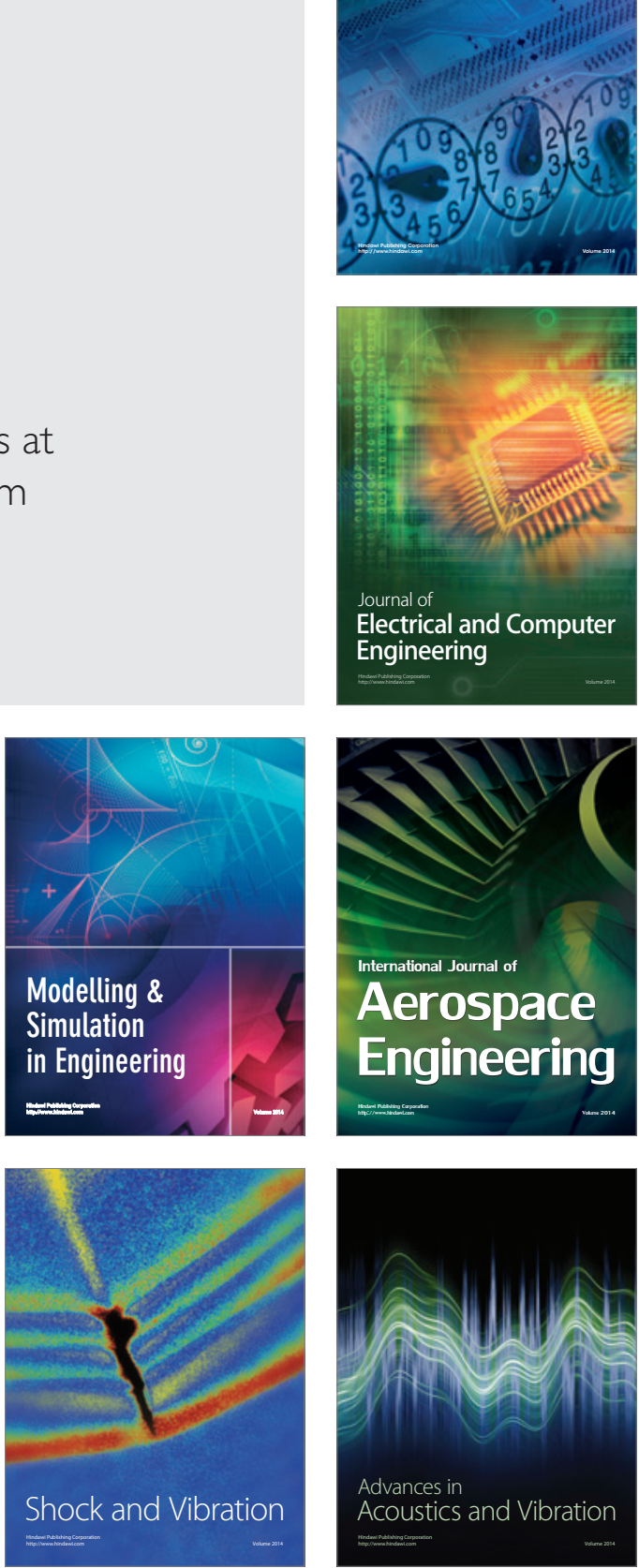